\title{
Karhunen-Loève expansion revisited for vector-valued random fields: scaling, errors and optimal basis.
}

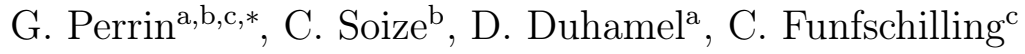 \\ ${ }^{a}$ Université Paris-Est, Laboratoire Navier (UMR 8205), CNRS, ENPC, IFSTTAR, \\ F-77455 Marne-la-Vallée. \\ ${ }^{b}$ Université Paris-Est, Modélisation et Simulation Multi-Échelle (MSME UMR 8208 \\ CNRS), 5 Bd. Descartes, 77454 Marne-la-Vallée, France. \\ ${ }^{c} S N C F$, Innovation and Research Department, Immeuble Lumière, 40 avenue des \\ Terroirs de France, 75611, Paris, Cedex 12, France.
}

\begin{abstract}
Due to scaling effects, when dealing with vector-valued random fields, the classical Karhunen-Loève expansion, which is optimal with respect to the total mean square error, tends to favorize the components of the random field that have the highest signal energy. When these random fields are to be used in mechanical systems, this phenomenon can introduce undesired biases for the results. This paper presents therefore an adaptation of the KarhunenLoève expansion that allows us to control these biases and to minimize them. This original decomposition is first analyzed from a theoretical point of view, and is then illustrated on a numerical example.
\end{abstract}

Keywords:

Karhunen-Loève expansion, vector-valued random field, optimal basis.

\section{Introduction}

For the last decades, the use of simulation has spread to every scientific and engineering fields, which has made possible the analysis of always more complex and refined physical models. While engineers were used to treating

\footnotetext{
*Corresponding author

Email addresses: guillaume.perrin@enpc.fr (G. Perrin), christian.soize@univ-paris-est.fr (C. Soize), denis.duhamel@enpc.fr (D. Duhamel), christine.funfschilling@sncf.fr (C. Funfschilling)
} 
static and unidimensional deterministic problems, they are currently more and more asked to solve three-dimensional dynamical stochastic problems. Nevertheless, in spite of the increasing computational power, the demand for computational ressources keeps exceeding strongly the actual supply. In this context, the use of innovative methodologies, such as reduction methods, still have a big role to play to sidestep direct approaches, in order to accurately estimate quantities of interest of complex systems.

The Karhunen-Loève (KL) expansion has therefore been used in many scientific fields to efficiently reduce the statistical complexity of random fields indexed over closed and bounded intervals, with continuous covariance func-

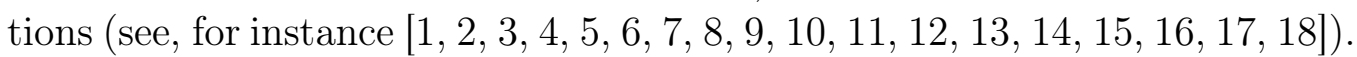
This expansion can then be coupled to a polynomial chaos expansion to completely characterize the distribution of random fields [19, 20, 21, 22, 23, 24, 25, 26, 27, 28, 29]. Mathematically, the KL expansion corresponds to the orthogonal projection theorem in separable Hilbert spaces, for which the Hilbertian basis is constructed as the eigenfunctions of the covariance operator of the random field of interest. The importance of this expansion stems from its optimality in the sense that, due to the orthogonal projection theorem in Hilbert spaces, it minimizes the total mean-squared error. In other words, for any integer $M$ and random fields $\boldsymbol{X}$, it can be extraced from the KL basis associated with $\boldsymbol{X}$ the $M$-dimension family that minimizes the total mean-squared error among all the $M$-dimension families that have been extracted from a countable Hilbertian basis.

When considering vector-valued random fields, this error can be written as a sum of weighted local errors, where the local errors and the weights are respectively the normalized mean-squared errors and the signal energies associated with each component of $\boldsymbol{X}$. Therefore, minimizing the total mean-squared error amounts to minimizing in priority the local errors corresponding to the components of $\boldsymbol{X}$ that have the highest signal energies. If the KL projection of the random field $\boldsymbol{X}$ is then used to propagate variability in mechanical systems, it has therefore to be kept in mind that the particular components of lowest signal energy will not necessary be realistic nor well characterized. If the quantities of interest of the studied system are however very dependent on a precise description of these components, such an optimal KL family may not be relevant and give biased results.

In this prospect, in addition to the classical mean-squared error, two local-global projection errors are introduced in this work: 
- $\varepsilon_{\boldsymbol{\beta}}^{2}$ corresponds to another weighted sum of local errors, for which weights are a priori or a posteriori chosen from sensitivity analysis;

- $\varepsilon_{\infty}^{2}$ refers to the maximal value of the local errors associated with each component of random field $\boldsymbol{X}$.

Indeed, these errors illustrate two classical expectations. On the first hand, error $\varepsilon_{\boldsymbol{\beta}}^{2}$ leads to projection families which are particularly adapted to the components of $\boldsymbol{X}$ of highest chosen weight. If the importance of each component of $\boldsymbol{X}$ for a given quantity of interest can be evaluated from a sensibility analysis, these weights can thus be chosen in order to maximize the relevance of the projection basis to analyze this chosen quantity of interest. One the other hand, if no information is available about the importance of each component of $\boldsymbol{X}$, making these weights be equal corresponds to the case where no component of $\boldsymbol{X}$ is favorized in the error to be minimized. In such a case, there is however no reason for the minimization of this equally weighted error to lead to a projection family for which each local error would be the same. This motivates thus the introduction of error $\varepsilon_{\infty}^{2}$, which forces us to search projection families, for which the description precision would be close for each component.

Based on an original scaled Karhunen-Loève expansion of $\boldsymbol{X}$, the idea of this work is therefore to propose a method to identify the optimal families that respectively minimize errors $\varepsilon_{\boldsymbol{\beta}}^{2}$ and $\varepsilon_{\infty}^{2}$.

In Section 2, the scaled Karhunen-Loève expansion is described. In particular, it will be shown how such a formalism allows the identification of the two former optimal basis to be constructed. Section 3 illustrates the possibilities of such an expansion on a practical application.

\section{Scaled Karhunen-Loève expansion and optimal basis for vector- valued random fields}

In this section, the definition of the two local-global errors $\varepsilon_{\boldsymbol{\beta}}^{2}$ and $\varepsilon_{\infty}^{2}$ is first presented. The proposed scaled KL expansion for vector-valued random fields is then introduced. It is finally shown in what extent such a decomposition can lead to the minimization of these two errors. 


\subsection{Local-global errors and optimal basis}

\subsubsection{Theoretical frame}

Let $(\Theta, \mathcal{C}, P)$ be a probability space. Let $\mathcal{H}=L^{2}\left(\Theta, \mathbb{R}^{Q}\right)$ be the space of all the second-order random vectors defined on $(\Theta, \mathcal{C}, P)$ with values in $\mathbb{R}^{Q}$, equipped with the inner product $\langle\cdot, \cdot\rangle$, such that for all $\boldsymbol{U}$ and $\boldsymbol{V}$ in $\mathcal{H}$,

$$
\langle\boldsymbol{U}, \boldsymbol{V}\rangle=\int_{\Theta} \boldsymbol{U}(\theta)^{T} \boldsymbol{V}(\theta) d P(\theta)=E\left[\boldsymbol{U}^{T} \boldsymbol{V}\right],
$$

where $E[\cdot]$ is the mathematical expectation. Let $\mathcal{P}(\Omega)$ be the space of all the second-order $\mathbb{R}^{Q}$-valued random fields, indexed by the compact interval $\Omega=[0, S]$, where $S<+\infty$. Let $\mathbb{H}=L^{2}\left(\Omega, \mathbb{R}^{Q}\right)$ be the space of square integrable functions on $\Omega$, with values in $\mathbb{R}^{Q}$, equipped with the inner product $(\cdot, \cdot)$, such that for all $\boldsymbol{u}$ and $\boldsymbol{v}$ in $\mathbb{H}$,

$$
(\boldsymbol{u}, \boldsymbol{v})=\int_{\Omega} \boldsymbol{u}(s)^{T} \boldsymbol{v}(s) d s .
$$

Let $\boldsymbol{X}=\{\boldsymbol{X}(s), s \in \Omega\}$ be an element of $\mathcal{P}(\Omega)$. Without loss of generality, it is supposed that the mean value of $\boldsymbol{X}$ is equal to zero:

$$
E[\boldsymbol{X}(s)]=\mathbf{0}, \forall s \in \Omega .
$$

The signal energy of $\boldsymbol{X}$, which corresponds to its $L_{2}$ norm, $\|\boldsymbol{X}\|_{2}$, can thus be written:

$$
\|\boldsymbol{X}\|_{2} \stackrel{\text { def }}{=} \sqrt{E[(\boldsymbol{X}, \boldsymbol{X})]}
$$

In the following, $\mathcal{F}^{(M)}=\left\{\boldsymbol{f}^{i}, 1 \leq i \leq M\right\}$ refers to a set of $M$ deterministic functions that has been extracted from any countable Hilbertian basis of $\mathbb{H}=L^{2}\left(\Omega, \mathbb{R}^{Q}\right)$. The projection of random field $\boldsymbol{X}$ on $\mathcal{F}^{(M)}$ is then written $\widehat{\boldsymbol{X}}^{(M)}$. The total normalized mean-squared errors associated with $\mathcal{F}^{(M)}$ is denoted as $\varepsilon^{2}\left(\mathcal{F}^{(M)}\right)$ and can thus be written as a sum of weighted local normalized mean-squared errors,

$$
\varepsilon_{q}^{2}\left(\mathcal{F}^{(M)}\right)=\frac{\left\|X_{q}-\widehat{X}_{q}^{(M)}\right\|_{2}^{2}}{\left\|X_{q}\right\|_{2}^{2}}, \quad 1 \leq q \leq Q,
$$

associated with each component $X_{q}$ of random field $\boldsymbol{X}$ : 


$$
\varepsilon^{2}\left(\mathcal{F}^{(M)}\right)=\sum_{q=1}^{Q}\left\{\frac{\left\|X_{q}\right\|_{2}^{2}}{\|\boldsymbol{X}\|_{2}^{2}}\right\} \varepsilon_{q}^{2}\left(\mathcal{F}^{(M)}\right) .
$$

\subsubsection{Optimality of the $K L$ expansion}

The matrix-valued covariance function, $\left[R_{X X}\right]$, of centered random field $\boldsymbol{X}$ is introduced as:

$$
\left[R_{X X}\left(s, s^{\prime}\right)\right]=E\left[\boldsymbol{X}(s) \otimes \boldsymbol{X}\left(s^{\prime}\right)\right], \forall\left(s, s^{\prime}\right) \in \Omega^{2} .
$$

It is assumed that $\left[R_{X X}\right]$ is square integrable on $\Omega \times \Omega$, that is to say

$$
\left\|\left[R_{X X}\right]\right\|_{\mathbb{M}}^{2} \stackrel{\text { def }}{=} \int_{\Omega} \int_{\Omega}\left\|\left[R_{X X}\left(s, s^{\prime}\right)\right]\right\|_{F}^{2} d s d s^{\prime}<+\infty,
$$

with $\|\cdot\|_{F}$ the Frobenius norm of matrices. The KL basis, $\mathcal{K}=\left\{\boldsymbol{k}^{i}, 1 \leq i\right\}$, associated with $\boldsymbol{X}$, can be constructed as a countable Hilbertian basis of $\mathbb{H}$, which is constituted of the eigenfunctions of covariance matrix-valued function $\left[R_{\boldsymbol{X} \boldsymbol{X}}\right]$, such that:

$$
\begin{aligned}
& \int_{\Omega}\left[R_{\boldsymbol{X} \boldsymbol{X}}\left(s, s^{\prime}\right)\right] \boldsymbol{k}^{i}\left(s^{\prime}\right) d s^{\prime}=\lambda_{i} \boldsymbol{k}^{i}(s), \quad s \in \Omega, \quad 1 \leq i, \\
& \left(\boldsymbol{k}^{i}, \boldsymbol{k}^{j}\right)=\delta_{i j}, \quad \lambda_{1} \geq \lambda_{2} \geq \cdots \rightarrow 0, \quad \sum_{i \geq 1} \lambda_{i}^{2}<+\infty,
\end{aligned}
$$

where $\delta_{i j}$ is the Kronecker symbol, equal to 1 if $i=j$ and to 0 otherwise (Issues concerning the solving of the integral eigenvalue problem, defined by Eq. (9), which is usually called Fredholm problem, can be found in [30, 31, 32]). Due to the orthogonal projection theorem in Hilbert space, it can thus be shown that for all $M \geq 1$, projection family $\mathcal{K}^{(M)}=\left\{\boldsymbol{k}^{i}, 1 \leq i \leq M\right\}$ is optimal in the sense that, for all family $\mathcal{F}^{(M)}$ :

$$
\varepsilon^{2}\left(\mathcal{K}^{(M)}\right) \leq \varepsilon^{2}\left(\mathcal{F}^{(M)}\right) .
$$

Let $\widetilde{X}^{(M)}$ be the projection of $\boldsymbol{X}$ on $\mathcal{K}^{(M)}$. Family $\mathcal{K}^{(M)}$ being orthonormal, it comes:

$$
\tilde{X}^{(M)}=\sum_{i=1}^{M} \sqrt{\lambda_{i}} \boldsymbol{k}^{i} \xi_{i}
$$


where $\boldsymbol{\xi}=\left(\xi_{1}, \cdots, \xi_{M}\right)$ is a centered random vector, for which components are uncorrelated and with variance equal to 1 . In particular, if $\boldsymbol{X}$ is a Gaussian random field, the components of $\boldsymbol{\xi}$ are normally distributed and statistically independent.

\subsubsection{Local-global errors}

From Eq. (6), minimizing $\varepsilon^{2}$ amounts therefore to minimizing in priority the local errors corresponding to the components of $\boldsymbol{X}$ that have the highest weights $\frac{\left\|X_{q}\right\|_{2}^{2}}{\|\boldsymbol{X}\|_{2}^{2}}$. In other words, for given values of $p, q$ and $M$, if $\left\|X_{p}\right\|_{2} \gg\left\|X_{q}\right\|_{2}$, the minimization of $\varepsilon^{2}$ can lead to the identification of a $M$-dimension truncated Karhunen-Loève family associated with $\boldsymbol{X}, \mathcal{K}^{(M)}$, such that $\varepsilon_{p}^{2}\left(\mathcal{K}^{(M)}\right) \ll \varepsilon_{q}^{2}\left(\mathcal{K}^{(M)}\right)$. Consequently, if $X_{p}$ and $X_{q}$ are independent, a two steps approach, based on the definition of two different families (one for $X_{p}$ and the components of $\boldsymbol{X}$ that depend on $X_{p}$, one for the other components of $\boldsymbol{X}$ that do not depend on $X_{p}$ ) would be more relevant. On the contrary, if $X_{p}$ and $X_{q}$ are indeed dependent, more elements have to be added in $\mathcal{K}^{(M)}$ to make $\varepsilon_{q}^{2}$ decrease, or another choice for the error function to be minimized has to be considered.

In this prospect, two local-global projection errors are introduced in this work, $\varepsilon_{\boldsymbol{\beta}}^{2}$ and $\varepsilon_{\infty}^{2}$, such that for any $\boldsymbol{\beta}$ in $] 0,+\infty\left[{ }^{Q}\right.$ :

$$
\begin{gathered}
\varepsilon_{\boldsymbol{\beta}}^{2}=\sum_{q=1}^{Q} \beta_{q}^{2} \varepsilon_{q}^{2}, \\
\varepsilon_{\infty}^{2}=\max _{1 \leq q \leq Q}\left\{\varepsilon_{q}^{2}\right\} .
\end{gathered}
$$

As presented in Section 1, if the reduction of the statistical complexity of random field $\boldsymbol{X}$ is carried out as a first step in a propagation of variability in mechanical systems, minimizing these two errors instead of error $\varepsilon^{2}$ should allow us to improve the relevance of the projection basis, whether the importance of each component of $\boldsymbol{X}$ for a given quantity of interest can be evaluated from a sensibility analysis or not.

\subsection{Scaled KL expansion}

Let $\boldsymbol{O}$ be an element of $\mathcal{S}^{(Q)}(1)=\{\boldsymbol{O} \in] 0,1\left[{ }^{Q}, \sum_{q=1}^{Q} O_{q}^{2}=1\right\}$. This allows us to define the scaled random field, $\boldsymbol{Y}(\boldsymbol{O})$, such that: 


$$
\begin{gathered}
\boldsymbol{Y}(\boldsymbol{O})=[\operatorname{Diag}(\boldsymbol{O})] \boldsymbol{X} \\
{[\operatorname{Diag}(\boldsymbol{O})]=\left[\begin{array}{cccc}
O_{1} & 0 & \cdots & 0 \\
0 & O_{2} & \ddots & \vdots \\
\vdots & \ddots & \ddots & 0 \\
0 & \cdots & 0 & O_{Q}
\end{array}\right] .}
\end{gathered}
$$

The autocorrelation function, $\left[R_{\boldsymbol{Y} \boldsymbol{Y}}(\boldsymbol{O})\right]$, of $\boldsymbol{Y}(\boldsymbol{O})$ is thus equal to:

$$
\left[R_{\boldsymbol{Y} \boldsymbol{Y}}(\boldsymbol{O})\right]=[\operatorname{Diag}(\boldsymbol{O})]\left[R_{\boldsymbol{X} \boldsymbol{X}}\right][\operatorname{Diag}(\boldsymbol{O})] .
$$

The family $\mathcal{K}^{(M)}(\boldsymbol{O})=\left\{\boldsymbol{k}^{i}(\boldsymbol{O}), 1 \leq i\right\}$ is thus denoted as the KarhunenLoève family associated with random field $\boldsymbol{Y}(\boldsymbol{O})$, such that:

$$
\begin{gathered}
\boldsymbol{Y}(\boldsymbol{O})=\sum_{i=1}^{+\infty} \boldsymbol{k}^{i}(\boldsymbol{O}) \sqrt{\lambda_{i}(\boldsymbol{O})} \xi_{i}(\boldsymbol{O}), \\
\lambda_{i}(\boldsymbol{O})=\left\langle\left(\boldsymbol{Y}(\boldsymbol{O}), \boldsymbol{k}^{i}(\boldsymbol{O})\right),\left(\boldsymbol{Y}(\boldsymbol{O}), \boldsymbol{k}^{i}(\boldsymbol{O})\right)\right\rangle, \quad \xi_{i}(\boldsymbol{O})=\frac{\left(\boldsymbol{Y}(\boldsymbol{O}), \boldsymbol{k}^{i}(\boldsymbol{O})\right)}{\sqrt{\lambda_{i}(\boldsymbol{O})}} .
\end{gathered}
$$

where it is reminded that, by construction, familly $\mathcal{K}^{(M)}(\boldsymbol{O})$ is orthonormal in $\mathbb{H}$, and projection coefficients $\left\{\xi_{i}(\boldsymbol{O}), i \geq 1\right\}$ are uncorrelated:

$$
\left(\boldsymbol{k}^{i}(\boldsymbol{O}), \boldsymbol{k}^{j}(\boldsymbol{O})\right)=E\left[\xi_{i}(\boldsymbol{O}) \xi_{j}(\boldsymbol{O})\right]=\delta_{i j}, \quad 1 \leq i, j .
$$

Since $O_{q} \neq 0$ for all $1 \leq q \leq Q$, matrix $[\operatorname{Diag}(\boldsymbol{O})]$ is invertible. Therefore, the projection of random field $\boldsymbol{X}$ on family $\mathcal{K}^{(M)}(\boldsymbol{O})$, that is denoted as $\widehat{\boldsymbol{X}}^{(M)}(\boldsymbol{O})$, is given by:

$$
\widehat{\boldsymbol{X}}^{(M)}(\boldsymbol{O})=\sum_{i=1}^{M}[\operatorname{Diag}(\boldsymbol{O})]^{-1} \boldsymbol{k}^{i}(\boldsymbol{O}) \sqrt{\lambda_{i}(\boldsymbol{O})} \xi_{i}(\boldsymbol{O}), \quad 1 \leq M
$$

The elements of $\mathcal{K}^{(M)}(\boldsymbol{O})$ are once again ordered such that the variance of the projection random variables are sorted in a decreasing order:

$$
\lambda_{1}(\boldsymbol{O}) \geq \lambda_{2}(\boldsymbol{O}) \geq \cdots \rightarrow 0 .
$$


According to Eqs. (5) and (66), for all $1 \leq M$, we finally have:

$$
\begin{gathered}
\varepsilon_{q}^{2}\left(\mathcal{K}^{(M)}(\boldsymbol{O})\right)=1-\frac{O_{q}^{-2}}{\left\|X_{q}\right\|_{2}^{2}} \sum_{i=1}^{M} \lambda_{i}(\boldsymbol{O}) \int_{\Omega}\left\{k_{q}^{i}(\boldsymbol{O}, s)\right\}^{2} d s, \quad 1 \leq q \leq Q \\
\varepsilon^{2}\left(\mathcal{K}^{(M)}(\boldsymbol{O})\right)=1-\frac{1}{\|\boldsymbol{X}\|_{2}^{2}} \sum_{q=1}^{Q} O_{q}^{-2} \sum_{i=1}^{M} \lambda_{i}(\boldsymbol{O}) \int_{\Omega}\left\{k_{q}^{i}(\boldsymbol{O}, s)\right\}^{2} d s .
\end{gathered}
$$

It can be verified that if $\boldsymbol{O}=\frac{1}{\sqrt{Q}}(1, \cdots, 1)$, the scaled KL expansion coincides with the classical and direct $\mathrm{KL}$ expansion associated with $\boldsymbol{X}$, defined in Section 2.1.2.

\subsection{Properties of the scaled KL expansion}

This section aims at emphasizing the main properties of the scaled KL expansion, on which the minimization of local-global errors $\varepsilon_{\boldsymbol{\beta}}^{2}$ and $\varepsilon_{\infty}^{2}$ will be based. First, the continuity of the applications $\boldsymbol{O} \mapsto \varepsilon_{\boldsymbol{\beta}}^{2}\left(\mathcal{K}^{(M)}(\boldsymbol{O})\right)$ and $\boldsymbol{O} \mapsto \varepsilon_{\infty}^{2}\left(\mathcal{K}^{(M)}(\boldsymbol{O})\right)$ on $S^{(Q)}(1)$ will be shown. Then, the mechanisms induced by the scaled KL expansion and its optimality are presented.

Lemma 1. Random field $\boldsymbol{Y}(\boldsymbol{O})$ and its realizations are continuous with respect to $\boldsymbol{O}$ and the $L_{2}$ norm on $S^{(Q)}(1)$.

Proof: Let $\boldsymbol{O}$ and $\boldsymbol{O}^{*}$ be two elements of $\mathcal{S}^{(Q)}(1)$.

1. We have:

$$
\begin{aligned}
\left\|\boldsymbol{Y}(\boldsymbol{O})-\boldsymbol{Y}\left(\boldsymbol{O}^{*}\right)\right\|_{2}^{2} & =\sum_{q=1}^{Q}\left(O_{q}-O_{q}^{*}\right)^{2}\left\|X_{q}\right\|_{2}^{2}, \\
& \leq C_{\boldsymbol{Y}}\left\|\boldsymbol{O}-\boldsymbol{O}^{*}\right\|_{\mathbb{R}^{Q}}^{2}
\end{aligned}
$$

where $\|\cdot\|_{\mathbb{R}^{Q}}$ is the Euclidian norm on $\mathbb{R}^{Q}$ and $C_{\boldsymbol{Y}}=\max _{1 \leq q \leq Q}\left\|X_{q}\right\|_{2}^{2}$ is a positive constant that is independent of $\boldsymbol{O}$ and $\boldsymbol{O}^{*}$. The application $\boldsymbol{O} \mapsto \boldsymbol{Y}(\boldsymbol{O})$ is therefore continuous on $\mathcal{S}^{(Q)}(1)$ with respect to the $L_{2}$ norm. 
2. In the same manner, let $\boldsymbol{X}(\theta)$ be a realization of $\boldsymbol{X}$, such that, by construction, $\boldsymbol{Y}(\boldsymbol{O}, \theta)=[\operatorname{Diag}(\boldsymbol{O})] \boldsymbol{X}(\theta)$ and $\boldsymbol{Y}\left(\boldsymbol{O}^{*}, \theta\right)=\left[\operatorname{Diag}\left(\boldsymbol{O}^{*}\right)\right] \boldsymbol{X}(\theta)$ are the corresponding realizations of $\boldsymbol{Y}(\boldsymbol{O})$ and $\boldsymbol{Y}\left(\boldsymbol{O}^{*}\right)$ respectively. Therefore:

$$
\begin{aligned}
\left\|\boldsymbol{Y}(\boldsymbol{O}, \theta)-\boldsymbol{Y}\left(\boldsymbol{O}^{*}, \theta\right)\right\|_{L_{2}}^{2} & \stackrel{\text { def }}{=}\left(\boldsymbol{Y}(\boldsymbol{O}, \theta)-\boldsymbol{Y}\left(\boldsymbol{O}^{*}, \theta\right), \boldsymbol{Y}(\boldsymbol{O}, \theta)-\boldsymbol{Y}\left(\boldsymbol{O}^{*}, \theta\right)\right) \\
& \leq\left\|\boldsymbol{O}-\boldsymbol{O}^{*}\right\|_{\mathbb{R}^{Q}}^{2}\left[\max _{1 \leq q \leq Q}\left\{\left(X_{q}(\theta), X_{q}(\theta)\right)\right\}\right]
\end{aligned}
$$

As $\max _{1 \leq q \leq Q}\left\{\left(X_{q}(\theta), X_{q}(\theta)\right)\right\}$ is a positive constant that is independent of $\boldsymbol{O}$ and $\boldsymbol{O}^{*}$, the application $\boldsymbol{O} \mapsto \boldsymbol{Y}(\boldsymbol{O}, \theta)$ is continuous on $\mathcal{S}^{(Q)}(1)$ with respect to the norm $\|\cdot\|_{L_{2}}$.

Equation (18) and Lemma 1 1yield that for any values of the set of random variables $\left\{\xi_{i}(\boldsymbol{O}), 1 \leq i\right\}$, whose mean values are equal to zero and variances are equal to one, the application $\boldsymbol{O} \mapsto \sum_{1 \leq i} \sqrt{\lambda_{i}(\boldsymbol{O})} \boldsymbol{k}^{i}(\boldsymbol{O}) \xi_{i}(\boldsymbol{O})$ is continuous on $S^{(Q)}(1)$ with respect to the $L_{2}$ norm. This motivates the introduction of the following hypothesis, that will be required for the next propositions to be valid.

Hypothesis 1 . For all $1 \leq i$, the applications $\boldsymbol{O} \mapsto \sqrt{\lambda_{i}(\boldsymbol{O})} \boldsymbol{k}^{i}(\boldsymbol{O})$ are supposed to be continuous on $S^{(Q)}(1)$ with respect to the norm $\|\cdot\|_{L_{2}}$.

Proposition 1. Under Hypothesis 1, the applications $\boldsymbol{O} \mapsto \varepsilon_{q}^{2}\left(\mathcal{K}^{(M)}(\boldsymbol{O})\right)$ are continuous with respect to the Euclidian norm on $S^{(Q)}(1)$, for all $1 \leq q \leq Q$.

Proof: If Hypothesis 1 is verified, due to the continuity properties of the product, of the sum, and of the integral over a closed interval, it can be deduced that for all $1 \leq M$,

$$
\boldsymbol{O} \mapsto \sum_{i=1}^{M} \lambda_{i}(\boldsymbol{O}) \int_{\Omega}\left\{k_{q}^{i}(\boldsymbol{O}, s)\right\}^{2} d s, \quad 1 \leq q \leq Q,
$$

are continuous with respect to the Euclidian norm on $S^{(Q)}(1)$. According to Eq. (23), this leads us to the continuity on $S^{(Q)}(1)$ of the applications $\boldsymbol{O} \mapsto \varepsilon_{q}^{2}\left(\mathcal{K}^{(M)}(\boldsymbol{O})\right)$, for all $1 \leq q \leq Q$ 
Corrolary 1. Under Hypothesis 1, the applications $\boldsymbol{O} \mapsto \varepsilon_{\boldsymbol{\beta}}^{2}\left(\mathcal{K}^{(M)}(\boldsymbol{O})\right)$ and $\boldsymbol{O} \mapsto \varepsilon_{\infty}^{2}\left(\mathcal{K}^{(M)}(\boldsymbol{O})\right)$ are continuous with respect to the Euclidian norm on $S^{(Q)}(1)$.

Proof: By construction of errors $\varepsilon_{\boldsymbol{\beta}}^{2}$ and $\varepsilon_{\infty}^{2}$, defined by Eqs. (13) and (14), this corrolary is a direct consequence of Proposition 1 .

Proposition 2. Under Hypothesis 1, for all $1 \leq M$, application $\boldsymbol{O} \mapsto$ $\varepsilon_{\infty}^{2}\left(\mathcal{K}^{(M)}(\boldsymbol{O})\right)$ admits a minimal value, $\boldsymbol{O}_{\infty}^{(M)}$, in $\mathcal{S}^{(Q)}(1)$.

Proof: Under Hypothesis 1, Corrolary 1 yields that application $\boldsymbol{O} \mapsto$ $\varepsilon_{\infty}^{2}\left(\mathcal{K}^{(M)}(\boldsymbol{O})\right)$ is continuous with respect to the Euclidian norm on $\mathcal{S}^{(Q)}(1)$ for all $1 \leq M$. It admits therefore a minimal value in any closed subset $\widehat{\mathcal{S}}(\epsilon)=\left\{\widehat{\boldsymbol{O}} \in[\epsilon, 1-\epsilon]^{Q}, \sum_{q=1}^{Q} O_{q}^{2}=1\right\}$, for all $0<\epsilon<1$.

Then, for $1 \leq q \leq Q$, if $O_{q}$ tends to zero, it can be noticed that $\varepsilon_{q}^{2}\left(\mathcal{K}^{(M)}(\boldsymbol{O})\right)$ tends to its maximal value as the weight of $X_{q}$ in the global minimization is almost zero. This leads us to the fact that it exists $0<\epsilon^{*}<1$ sufficiently small, such that for all $\boldsymbol{O}$ and $\boldsymbol{O}^{*}$ in $\widehat{\mathcal{S}}\left(\epsilon^{*}\right)$ and $\mathcal{S}^{(Q)}(1) \backslash \widehat{\mathcal{S}}\left(\epsilon^{*}\right)$ respectively, $\varepsilon_{\infty}^{2}(\boldsymbol{O}) \leq \varepsilon_{\infty}^{2}\left(\boldsymbol{O}^{*}\right)$. In other words, it exists $\epsilon^{*}$ in $] 0,1\left[\right.$ and $\boldsymbol{O}_{\infty}^{(M)}$ in $\mathcal{S}^{(Q)}(1)$ such that:

$$
\boldsymbol{O}_{\infty}^{(M)}=\arg \min _{\boldsymbol{O} \in \hat{\mathcal{S}}\left(\epsilon^{*}\right)}\left\{\varepsilon_{\infty}^{2}(\boldsymbol{O})\right\}=\arg \min _{\boldsymbol{O} \in \mathcal{S}^{(Q)}(1)}\left\{\varepsilon_{\infty}^{2}(\boldsymbol{O})\right\}
$$

The importance of such a vector $\boldsymbol{O}_{\infty}^{(M)}$ for the minimization of error $\varepsilon_{\infty}^{2}$ will be discussed in Section 2.5. Although the perturbation of $\left[R_{\boldsymbol{X} \boldsymbol{X}}\right]$, defined by Eq. (17), is quadratic with respect to vector $\boldsymbol{O}$, there is no theoretical result in the pertubation theory field that could guarantee the validity of Hypothesis 1 in the general case. From a discrete point of view, applications $\boldsymbol{O} \mapsto \sqrt{\lambda_{i}(\boldsymbol{O})} \boldsymbol{k}^{i}(\boldsymbol{O})$ can however always be considered as continuous, as for any discontinuous application $\mathcal{A}$, it exists a continuous application $\mathcal{A}^{*}$, such that the projections of $\mathcal{A}$ and $\mathcal{A}^{*}$ on the same discretized space are the same. Hence, in the following, it is supposed that we are within the framework of Hypothesis 1 ,

The next Lemma and Proposition aim now at emphasizing how the scaled KL expansion could be used to favorize or defavorize on purpose the characterization of a particular component of $\boldsymbol{X}$. 
Lemma 2. For all $\boldsymbol{O}$ in $\mathcal{S}^{(Q)}(1)$ and for all $\mathcal{F}^{(M)}$ in $\mathbb{H}^{M}$, we have:

$$
\sum_{q=1}^{Q} O_{q}^{2}\left\|X_{q}\right\|_{2}^{2} \varepsilon_{q}^{2}\left(\mathcal{K}^{(M)}(\boldsymbol{O})\right) \leq \sum_{q=1}^{Q} O_{q}^{2}\left\|X_{q}\right\|_{2}^{2} \varepsilon_{q}^{2}\left(\mathcal{F}^{(M)}\right) .
$$

Proof: The proof of this lemma is detailed in AppendixA.

In other words, Lemma 2 underlines that for all $\boldsymbol{O}$ in $\mathcal{S}^{(Q)}(1)$, family $\mathcal{K}^{(M)}(\boldsymbol{O})$ is $M$-optimal for $\boldsymbol{X}$ regarding error $\sum_{q=1}^{Q} O_{q}^{2}\left\|X_{q}\right\|_{2}^{2} \varepsilon_{q}^{2}$. For $1 \leq$ $p \neq q \leq Q$, imposing $O_{p}^{2}\left\|X_{p}\right\|_{2}^{2}>O_{q}^{2}\left\|X_{q}\right\|_{2}^{2}$ tends therefore to favorize the characterization of $X_{p}$ rather than the one of $X_{q}$. This can be seen from the following proposition:

Proposition 3. For any $\boldsymbol{O}=\left(O_{1}, \cdots, O_{q}\right)$ in $\mathcal{S}^{(Q)}(1)$ and for all $\kappa$ such that $0<\kappa<\left\{\sum_{q=1}^{Q-1} O_{q}^{2}\right\}^{-1 / 2}$, the vector $\boldsymbol{O}^{*}=\left(\kappa O_{1}, \cdots, \kappa O_{Q-1}, \sqrt{1-\kappa^{2} \sum_{q=1}^{Q-1} O_{q}^{2}}\right)$ is in $\mathcal{S}^{(Q)}(1)$. For $\kappa=1$, we have $\boldsymbol{O}=\boldsymbol{O}^{*}$ and $\kappa$ can be smaller or larger than 1. We then have:

$$
\left\{\varepsilon_{Q}^{2}\left(\mathcal{K}^{(M)}\left(\boldsymbol{O}^{*}\right)\right)-\varepsilon_{Q}^{2}\left(\mathcal{K}^{(M)}(\boldsymbol{O})\right)\right\}\left\{\kappa^{2}-1\right\} \geq 0
$$

\section{Proof:}

1. If $\boldsymbol{O}=\left(O_{1}, \cdots, O_{Q}\right)$ is in $\mathcal{S}^{(Q)}(1)$, then $\sum_{q=1}^{Q} O_{q}^{2}=1$. Hence, if $0<\kappa<$ $\left\{\sum_{q=1}^{Q-1} O_{q}^{2}\right\}^{-1 / 2}, \sum_{q=1}^{Q}\left(O_{q}^{*}\right)^{2}=1$, which shows that $\boldsymbol{O}^{*}$ is in $\mathcal{S}^{(Q)}(1)$.

2. Moreover, Lemma 2 yields:

$$
\left\{\begin{array}{l}
\sum_{q=1}^{Q} O_{q}^{2}\left\|X_{q}\right\|_{2}^{2} \varepsilon_{q}^{2}\left(\mathcal{K}^{(M)}(\boldsymbol{O})\right) \leq \sum_{q=1}^{Q} O_{q}^{2}\left\|X_{q}\right\|_{2}^{2} \varepsilon_{q}^{2}\left(\mathcal{K}^{(M)}\left(\boldsymbol{O}^{*}\right)\right) \\
\sum_{q=1}^{Q}\left(O_{q}^{*}\right)^{2}\left\|X_{q}\right\|_{2}^{2} \varepsilon_{q}^{2}\left(\mathcal{K}^{(M)}\left(\boldsymbol{O}^{*}\right)\right) \leq \sum_{q=1}^{Q}\left(O_{q}^{*}\right)^{2}\left\|X_{q}\right\|_{2}^{2} \varepsilon_{q}^{2}\left(\mathcal{K}^{(M)}(\boldsymbol{O})\right)
\end{array}\right.
$$

which can, for all $c_{a}$ and $c_{b}$ in $\mathbb{R}^{+}$, be written in a more compact form as:

$$
\sum_{q=1}^{Q}\left\|X_{q}\right\|_{2}^{2}\left\{\varepsilon_{q}^{2}\left(\mathcal{K}^{(M)}\left(\boldsymbol{O}^{*}\right)\right)-\varepsilon_{q}^{2}\left(\mathcal{K}^{(M)}(\boldsymbol{O})\right)\right\}\left\{c_{a} O_{q}^{2}-c_{b}\left(O_{q}^{*}\right)^{2}\right\} \geq 0 .
$$


Choosing $c_{b}=1$ and $c_{a}=\kappa^{2}$ yields:

$$
\left\{\varepsilon_{Q}^{2}\left(\mathcal{K}^{(M)}\left(\boldsymbol{O}^{*}\right)\right)-\varepsilon_{Q}^{2}\left(\mathcal{K}^{(M)}(\boldsymbol{O})\right)\right\}\left\{\kappa^{2}-1\right\} \geq 0
$$

Hence, if $\kappa \geq 1$, that is to say if the weights of all components of $\boldsymbol{X}$, but the one of $X_{Q}$, have been increased in the choice of $\boldsymbol{O}^{*}$, the projection of $X_{Q}$ on $\mathcal{K}^{(M)}\left(\boldsymbol{O}^{*}\right)$ will be less precise than its projection on $\mathcal{K}^{(M)}(\boldsymbol{O})$ because $\varepsilon_{Q}^{2}\left(\mathcal{K}^{(M)}\left(\boldsymbol{O}^{*}\right)\right) \geq \varepsilon_{Q}^{2}\left(\mathcal{K}^{(M)}(\boldsymbol{O})\right)$. On the contrary, if $\kappa \leq 1$, the weight of $X_{Q}$ in the scaled KL expansion defined in Section 2.2 is increased by comparison to the other components of $\boldsymbol{X}$, such that the projection of $X_{Q}$ on $\mathcal{K}^{(M)}\left(\boldsymbol{O}^{*}\right)$ will be better than its projection on $\mathcal{K}^{(M)}(\boldsymbol{O})$ because $\varepsilon_{Q}^{2}\left(\mathcal{K}^{(M)}\left(\boldsymbol{O}^{*}\right)\right) \leq$ $\varepsilon_{Q}^{2}\left(\mathcal{K}^{(M)}(\boldsymbol{O})\right)$.

By playing on the values of the components of $\boldsymbol{O}$, the scaled KL expansion thus appears to be able to favorize or defavorize on purpose the characterization of a particular component of $\boldsymbol{X}$. The goal of the next sections is therefore to define a method to minimize errors $\varepsilon_{\boldsymbol{\beta}}^{2}$ and $\varepsilon_{\infty}^{2}$, based on this scaled KL expansion.

\subsection{Minimization of a weighted sum of local errors}

The minimization of error $\varepsilon_{\boldsymbol{\beta}}^{2}$, defined by Eq. (13), is a direct consequence of Lemma 2, Indeed, for all $\boldsymbol{\beta}$ in $\mathcal{S}^{(Q)}(1)$, it can directly be seen that the choice

$$
O_{q}^{\boldsymbol{\beta}}=\frac{\beta_{q}}{\left\|X_{q}\right\|_{2}}, \quad 1 \leq q \leq Q
$$

leads us to the minimization of error $\varepsilon_{\boldsymbol{\beta}}^{2}$, such that:

$$
\mathcal{K}^{(M)}\left(\boldsymbol{O}^{\boldsymbol{\beta}}\right)=\arg \min _{\mathcal{F}(M) \in \mathbb{H}^{M}}\left\{\varepsilon_{\boldsymbol{\beta}}^{2}\left(\mathcal{F}^{(M)}\right)\right\}, \quad 1 \leq M .
$$

Hence, just by considering the KL expansion of $\boldsymbol{Y}(\boldsymbol{O})=[\operatorname{Diag}(\boldsymbol{O})] \boldsymbol{X}$ rather than $\boldsymbol{X}$, it is possible to construct projection families that could favorize particular components of $\boldsymbol{X}$, from a priori or a posteriori choices for $\boldsymbol{\beta}$. 


\subsection{Minimization of the maximal value of the local errors}

The optimal families $\mathcal{F}_{\infty}^{(M)}$, which minimize error $\varepsilon_{\infty}^{2}$, that is to say such that:

$$
\mathcal{F}_{\infty}^{(M)}=\arg \min _{\mathcal{F}^{(M)} \in \mathbb{H}^{M}}\left\{\varepsilon_{\infty}^{2}\left(\mathcal{F}^{(M)}\right)\right\}=\arg \min _{\mathcal{F}^{(M)} \in \mathbb{H}^{M}}\left\{\max _{1 \leq q \leq Q} \varepsilon_{q}^{2}\left(\mathcal{F}^{(M)}\right)\right\},
$$

have been introduced to minimize the local errors associated with each component of $\boldsymbol{X}$. These projection families stem however from a Min-Max optimization on the very large space $\mathbb{H}^{M}$, such that their direct numerical identification can be very difficult. As the dimension of $\mathcal{S}^{(Q)}(1)$ is comparatively very small, the idea presented in this section is thus to use the former scaled KL expansion, defined in Section [2.2, to approximate $\mathcal{F}_{\infty}^{(M)}$ as the solution of an optimization problem with respect to $\boldsymbol{O}$ in $\mathcal{S}^{(Q)}(1)$, rather than an optimization problem with respect to $\mathcal{F}^{(M)}$ in $\mathbb{H}^{M}$. For all $1 \leq M$, we thus define $\mathcal{K}^{(M)}\left(\boldsymbol{O}_{\infty}^{(M)}\right)$ as the scaled KL basis associated with the vector $\boldsymbol{O}_{\infty}^{(M)}$, such that:

$$
\boldsymbol{O}_{\infty}^{(M)}=\arg \min _{\boldsymbol{O} \in \mathcal{S}^{(Q)}(1)}\left\{\varepsilon_{\infty}^{2}(\boldsymbol{O})\right\}
$$

for which existence stems from Proposition 2 ,

Whereas vector $\boldsymbol{O}^{\boldsymbol{\beta}}$, defined by Eq. (34), is independent of $M$, it has to be reminded that vector $\boldsymbol{O}_{\infty}^{(M)}$ depends on $M$ in the general case.

This section aims first at quantifying the distance between $\mathcal{K}^{(M)}\left(\boldsymbol{O}_{\infty}^{(M)}\right)$ and $\mathcal{F}_{\infty}^{(M)}$. In the two dimensional case $(Q=2)$, it will be shown in particular that $\mathcal{K}^{(M)}\left(\boldsymbol{O}_{\infty}^{(M)}\right)=\mathcal{F}_{\infty}^{(M)}$. At last, based on Proposition 3 , an algorithm to numerically solve Eq. (37) is presented.

2.5.1. Quantification of the error introduced by the approximated identification problem

Lemma 3. For all $M \geq 1$ and for all $\boldsymbol{O}$ in $\mathcal{S}^{(Q)}(1)$, the relevance of $\mathcal{K}^{(M)}(\boldsymbol{O})$ to minimize error $\varepsilon_{\infty}^{2}$ can be assessed as:

$$
0 \leq \varepsilon_{\infty}^{2}\left(\mathcal{K}^{(M)}(\boldsymbol{O})\right)-\varepsilon_{\infty}^{2}\left(\mathcal{F}_{\infty}^{(M)}\right) \leq \mathcal{U B}(\boldsymbol{O})
$$


where:

$$
\begin{gathered}
\mathcal{U B}(\boldsymbol{O}) \stackrel{\text { def }}{=} \frac{\sum_{q=1}^{Q} O_{q}^{2}\left\|X_{q}\right\|_{2}^{2} \delta_{q}^{2}\left(\mathcal{K}^{(M)}(\boldsymbol{O})\right)}{\sum_{q=1}^{Q} O_{q}^{2}\left\|X_{q}\right\|_{2}^{2}} \\
0 \leq \delta_{q}^{2}\left(\mathcal{K}^{(M)}(\boldsymbol{O})\right) \stackrel{\text { def }}{=} \varepsilon_{\infty}^{2}\left(\mathcal{K}^{(M)}(\boldsymbol{O})\right)-\varepsilon_{q}^{2}\left(\mathcal{K}^{(M)}(\boldsymbol{O})\right), \quad 1 \leq q \leq Q .
\end{gathered}
$$

$\square$ Proof: The first inequality $\varepsilon_{\infty}^{2}\left(\mathcal{K}^{(M)}(\boldsymbol{O})\right) \geq \varepsilon_{\infty}^{2}\left(\mathcal{F}_{\infty}^{(M)}\right)$ is a direct consequence of the optimality of $\mathcal{F}_{\infty}^{(M)}$. Let $\boldsymbol{O}$ be an element in $\mathcal{S}^{(Q)}(1)$. From Lemma 2, it can therefore be deduced that:

$$
\begin{aligned}
\sum_{q=1}^{Q} O_{q}^{2}\left\|X_{q}\right\|_{2}^{2} \varepsilon_{q}^{2}\left(\mathcal{K}^{(M)}(\boldsymbol{O})\right) & \leq \sum_{q=1}^{Q} O_{q}^{2}\left\|X_{q}\right\|_{2}^{2} \varepsilon_{q}^{2}\left(\mathcal{F}_{\infty}^{(M)}\right) \\
& \leq \varepsilon_{\infty}^{2}\left(\mathcal{F}_{\infty}^{(M)}\right)\left\{\sum_{q=1}^{Q} O_{q}^{2}\left\|X_{q}\right\|_{2}^{2}\right\}
\end{aligned}
$$

such that, by definition of $\left\{\delta_{1}^{2}\left(\mathcal{K}^{(M)}(\boldsymbol{O})\right), \cdots, \delta_{Q}^{2}\left(\mathcal{K}^{(M)}(\boldsymbol{O})\right)\right\}$ :

$$
\left\{\varepsilon_{\infty}^{2}\left(\mathcal{K}^{(M)}(\boldsymbol{O})\right)-\varepsilon_{\infty}^{2}\left(\mathcal{F}_{\infty}^{(M)}\right)\right\}\left\{\sum_{q=1}^{Q} O_{q}^{2}\left\|X_{q}\right\|_{2}^{2}\right\} \leq \sum_{q=1}^{Q} O_{q}^{2}\left\|X_{q}\right\|_{2}^{2} \delta_{q}^{2}\left(\mathcal{K}^{(M)}(\boldsymbol{O})\right),
$$

which proves the second part of the inequality.

This Lemma emphasizes that the closer the local errors are, the more relevant projection family $\mathcal{K}^{(M)}(\boldsymbol{O})$ is. In particular, quantity $\mathcal{U} \mathcal{B}\left(\boldsymbol{O}_{\infty}^{(M)}\right)$ defines an upper bound for the error introduced by the consideration of the approximated problem defined by Eq. (37). Lemma 3 leads us moreover to the following proposition:

Proposition 4. If the following equalities are verified:

$$
\varepsilon_{1}^{2}\left(\mathcal{K}^{(M)}\left(\boldsymbol{O}_{\infty}^{(M)}\right)\right)=\cdots=\varepsilon_{Q}^{2}\left(\mathcal{K}^{(M)}\left(\boldsymbol{O}_{\infty}^{(M)}\right)\right)
$$

then the family $\mathcal{K}^{(M)}\left(\boldsymbol{O}_{\infty}^{(M)}\right)$ minimizes $\varepsilon_{\infty}^{2}$. 
$\square$ Proof: By construction, if $\varepsilon_{1}^{2}\left(\mathcal{K}^{(M)}\left(\boldsymbol{O}_{\infty}^{(M)}\right)\right)=\cdots=\varepsilon_{Q}^{2}\left(\mathcal{K}^{(M)}\left(\boldsymbol{O}_{\infty}^{(M)}\right)\right)$, then $\delta_{1}^{2}\left(\mathcal{K}^{(M)}\left(\boldsymbol{O}_{\infty}^{(M)}\right)\right)=\cdots=\delta_{Q}^{2}\left(\mathcal{K}^{(M)}\left(\boldsymbol{O}_{\infty}^{(M)}\right)\right)=0$. Hence, from Lemma 3, we get $\varepsilon_{\infty}^{2}\left(\mathcal{K}^{(M)}\left(\boldsymbol{O}_{\infty}^{(M)}\right)\right)=\varepsilon_{\infty}^{2}\left(\mathcal{F}_{\infty}^{(M)}\right)$, such that $\mathcal{K}^{(M)}\left(\boldsymbol{O}_{\infty}^{(M)}\right)=\mathcal{F}_{\infty}^{(M)}$

\subsubsection{Identification of the optimal scaling vector}

By construction, it can directly be seen that, for all $\alpha$ in $\mathbb{R}, \mathcal{K}^{(M)}(\boldsymbol{O})=$ $\mathcal{K}^{(M)}(\alpha \boldsymbol{O})$. Hence, if the conditions of Proposition 4 are fulfilled, that is to say if $\varepsilon_{1}^{2}\left(\mathcal{K}^{(M)}\left(\boldsymbol{O}_{\infty}^{(M)}\right)\right)=\cdots=\varepsilon_{Q}^{2}\left(\mathcal{K}^{(M)}\left(\boldsymbol{O}_{\infty}^{(M)}\right)\right)$, the scaling vector $\boldsymbol{O}_{\infty}^{(M)}$ is solution of the following problem:

$$
\mathcal{K}^{(M)}(\boldsymbol{O})=\mathcal{K}^{(M)}\left([\operatorname{Diag}(\boldsymbol{O})] \boldsymbol{\epsilon}^{2}\left(\mathcal{K}^{(M)}(\boldsymbol{O})\right)\right),
$$

where the matrix $[\operatorname{Diag}(\boldsymbol{O})]$ is defined by Eq. (16), and where:

$$
\boldsymbol{\epsilon}^{2}\left(\mathcal{K}^{(M)}(\boldsymbol{O})\right)=\left(\varepsilon_{1}^{2}\left(\mathcal{K}^{(M)}(\boldsymbol{O})\right), \cdots, \varepsilon_{q}^{2}\left(\mathcal{K}^{(M)}(\boldsymbol{O})\right)\right) .
$$

This motivates the following iterative algorithm for the identification of scaling vector $\boldsymbol{O}_{\infty}^{(M)}$. For given parameters $\tau$ and $\gamma$ :

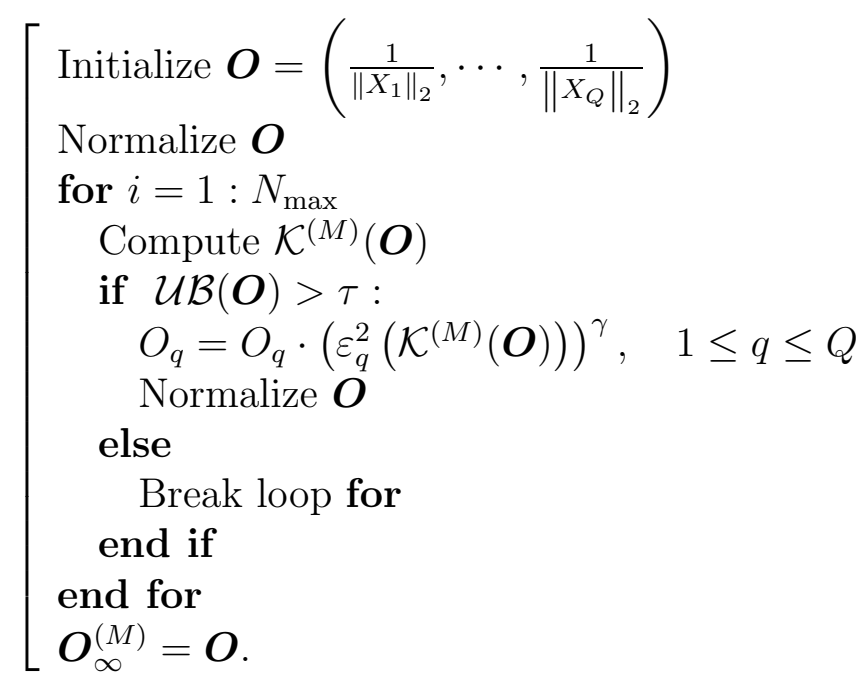

Parameter $\tau$ corresponds to the chosen precision of the numerical convergence, whereas $\gamma$ controls the speed of the convergence, and has to be adapted to avoid numerical instabilities. For our applications, $\gamma$ will be chosen equal to $1 / 2$. In such an algorithm, at each iteration $(n+1)$, the weight of $X_{q}$ in the KL expansion, $\left(O_{q}^{(n+1)}\right)^{2}\left\|X_{q}\right\|_{2}^{2}$, is updated with respect to the local error $\varepsilon_{q}^{2}\left(\mathcal{K}^{(M)}\left(\boldsymbol{O}^{(n)}\right)\right)$ of the former step. Hence, the weights of the less 
well characterized components of $\boldsymbol{X}$, for which local errors $\varepsilon_{q}^{2}\left(\mathcal{K}^{(M)}\left(\boldsymbol{O}^{(n)}\right)\right)$ are the highest at iteration $n$, will be increased the most at the new iteration $(n+1)$. In the general case, no convergence property for this algorithm has been proved yet, but under the following conditions:

$$
\lim _{O_{q}^{2} \rightarrow 1} \varepsilon_{q}^{2}\left(\mathcal{K}^{(M)}(\boldsymbol{O})\right) \leq \min _{1 \leq p \neq q \leq Q}\left\{\lim _{O_{q}^{2} \rightarrow 1} \varepsilon_{p}^{2}\left(\mathcal{K}^{(M)}(\boldsymbol{O})\right)\right\}, \quad 1 \leq q \leq Q
$$

it is assumed that the algorithm defined by Eq. (46) gives very promising results for the minimization of function $\boldsymbol{O} \mapsto \varepsilon_{\infty}^{2}\left(\mathcal{K}^{(M)}(\boldsymbol{O})\right)$ in a very few number of iterations. In other words, in cases where the weight of $X_{q}$ in the scaled KL expansion is much higher than the weights of the other components $\left\{X_{p}, 1 \leq p \neq q \leq Q\right\}$, if $X_{q}$ still remains badly characterized, then there is no reason for such an algorithm to converge to a satisfying result. In practice, these conditions are not very restrictive, and are most of the time verified for correlated vector-valued random fields.

In particular, under Hypothesis 1, when dealing with two dimensional cases $\left(Q=2, \boldsymbol{O}=\left(O_{1}, \sqrt{1-O_{1}^{2}}\right)\right)$, Propositions 11 and 3 yield that errors functions $O_{1} \mapsto \varepsilon_{1}^{2}\left(O_{1}\right)$ and $O_{1} \mapsto \varepsilon_{2}^{2}\left(O_{1}\right)$ are continuous and respectively decreases and increases with respect to $O_{1}$ in $] 0,1[$. Therefore, if conditions defined by Eq. (47) are fulfilled, it exists $\boldsymbol{O}_{\infty}^{(M)}$ in $\mathcal{S}^{(Q)}(1)$ such that $\varepsilon_{1}^{2}\left(\boldsymbol{O}_{\infty}^{(M)}\right)=\varepsilon_{2}^{2}\left(\boldsymbol{O}_{\infty}^{(M)}\right)$. Therefore, according to Proposition 4 , optimal basis $\mathcal{F}_{\infty}^{(M)}$ could be in these cases exactly identified from the solving of the optimization problem that is defined by Eq. (37).

\section{Application}

Most of the results emphasized in Section 2 are illustrated in this section on a practical example. This section is divided in three parts: first, a particular $\mathbb{R}^{4}$-valued random field is generated from its Karhunen-Loève expansion; then the influence of scaling vector $\boldsymbol{O}$ on the local errors is emphasized; at last, it is shown in what extent the scaled KL expansion allows us to identify optimal families $\mathcal{F}_{\infty}^{(M)}$ and $\mathcal{F}_{\boldsymbol{\beta}}^{(M)}$ for several values of $\boldsymbol{\beta}$ in $\mathcal{S}^{(Q)}(1)$ and any values of $M \geq 1$. 


\subsection{Generation of a vector-valued random field}

In this application, the dimension of random field $\boldsymbol{X}, Q$, is chosen equal to 4 , and $\Omega=[0,1]$. A particular matrix-valued covariance function, $\left[R_{\boldsymbol{X} \boldsymbol{X}}\right]$, is then postulated, for which some projections are represented in Figures 1 and 2. Random field $\boldsymbol{X}$, which is still supposed to be centered, can thus be written as:

$$
\boldsymbol{X}=\sum_{i=1}^{+\infty} \sqrt{\Lambda_{i}} \boldsymbol{k}^{i} \xi_{i}
$$

where, for all $i \geq 1$, couples $\left(\Lambda_{i}, \boldsymbol{k}^{i}\right)$ are solution of the Fredholm problem associated with $\left[R_{\boldsymbol{X} \boldsymbol{X}}\right]$ :

$$
\int_{\Omega}\left[R_{\boldsymbol{X} \boldsymbol{X}}\left(s, s^{\prime}\right)\right] \boldsymbol{k}^{i}\left(s^{\prime}\right) d s^{\prime}=\Lambda_{i} \boldsymbol{k}^{i}(s), \forall s \in \Omega,
$$

and coefficients $\left\{\xi_{i}, i \geq 1\right\}$ are uncorrelated random variables. For the sake of simplicity, these coefficients are moreover considered independent and normally distributed, which amounts to supposing that $\boldsymbol{X}$ is Gaussian. In particular, $\left[R_{\boldsymbol{X} \boldsymbol{X}}\right]$ has been chosen such that $\left\|X_{1}\right\|_{2}>\left\|X_{2}\right\|_{2}>\left\|X_{3}\right\|_{2}>\left\|X_{4}\right\|_{2}$. Further details about the generation of $\left[R_{\boldsymbol{X} \boldsymbol{X}}\right]$ can be seen in AppendixB. As an illustration, a particular realization, $\boldsymbol{X}(\theta)$, of $\boldsymbol{X}$ is represented in Figure 3. From Eq. (23), it is reminded that for any value of $\boldsymbol{O}$ in $\mathcal{S}^{(Q)}(1)$, for all $1 \leq q \leq Q$, and for all $M \geq 1$, errors $\varepsilon_{q}^{2}\left(\mathcal{K}^{(M)}(\boldsymbol{O})\right)$ can directly be computed by the scaled KL expansion.

\subsection{Influence of the scaling vector on the local errors}

According to Section 2, by introducing vector $\boldsymbol{O}=\left(O_{1}, O_{2}, O_{3}, O_{4}\right)$, we should be able to balance the values of local errors $\varepsilon_{q}^{2}$, for $1 \leq q \leq 4$. In particular, it has been shown in Section 2.3 that for $\boldsymbol{O}=\frac{1}{\sqrt{3+\kappa^{2}}}(1,1,1, \kappa)$ and for all $1 \leq M, \varepsilon_{4}^{2}\left(\mathcal{K}^{(M)}(\boldsymbol{O})\right)$ decreases with respect to $\kappa$ on $] 0,+\infty[$. Hence, if $\kappa$ tends to zero, $\varepsilon_{4}^{2}\left(\mathcal{K}^{(M)}(\boldsymbol{O})\right)$ is bound to converge to its maximal value, as the weight of $X_{4}$ in the minimization of $\sum_{q=1}^{4} O_{q}^{2}\left\|X_{q}\right\|_{2}^{2} \varepsilon_{q}^{2}$ becomes negligible. On the contrary, if $\kappa$ tends to infinity, $\varepsilon_{4}^{2}\left(\mathcal{K}^{(M)}(\boldsymbol{O})\right)$ will tend to its minimal value, as the minimization of $\sum_{q=1}^{4} O_{q}^{2}\left\|X_{q}\right\|_{2}^{2} \varepsilon_{q}^{2}$ will completely be driven by $\varepsilon_{4}^{2}$. This phenomenon can be seen in Figure 4, where the evolution of local errors $\varepsilon_{q}^{2}\left(\mathcal{K}^{(M)}(\boldsymbol{O})\right)$ with respect to $\kappa$ is represented. 

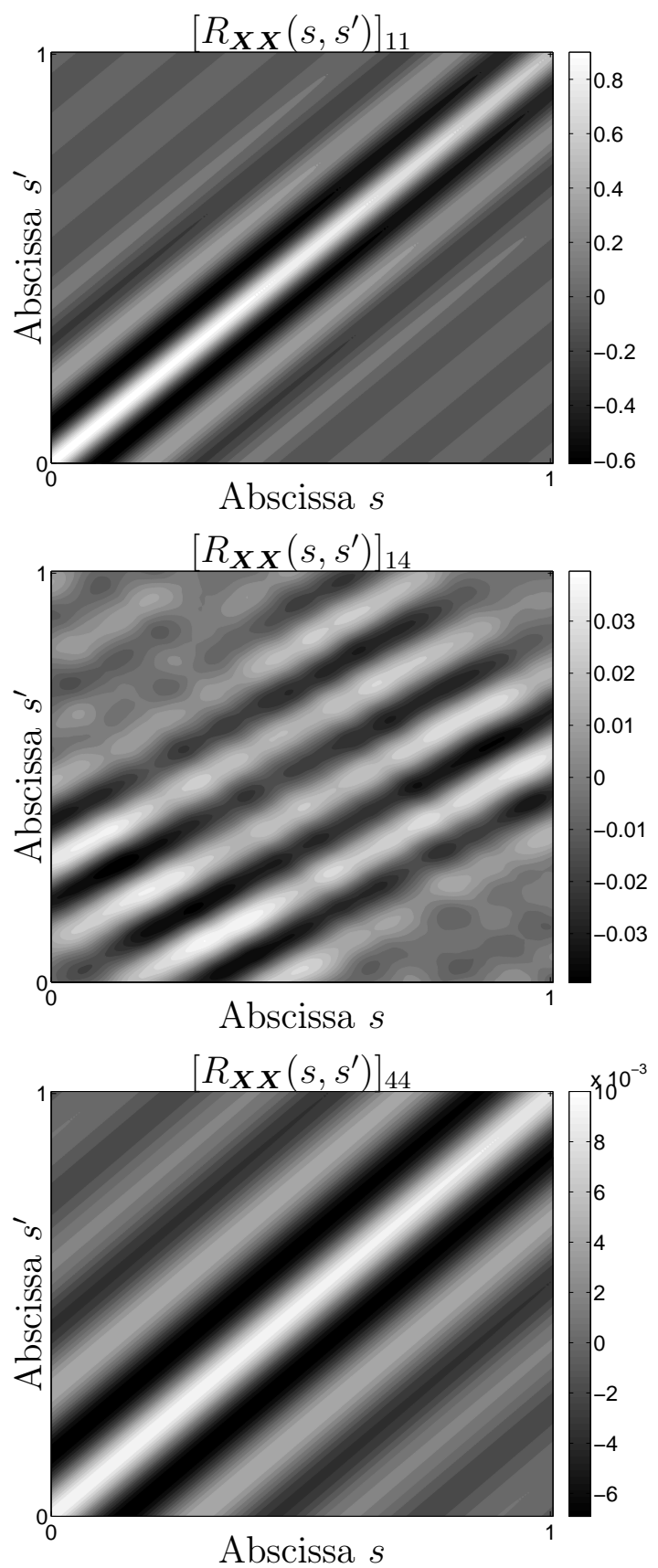

Figure 1: Representations of $\left[R_{\boldsymbol{X} \boldsymbol{X}}\right]_{11},\left[R_{\boldsymbol{X} \boldsymbol{X}}\right]_{14}$ and $\left[R_{\boldsymbol{X} \boldsymbol{X}}\right]_{44}$ on $[0,1] \times[0,1]$. 


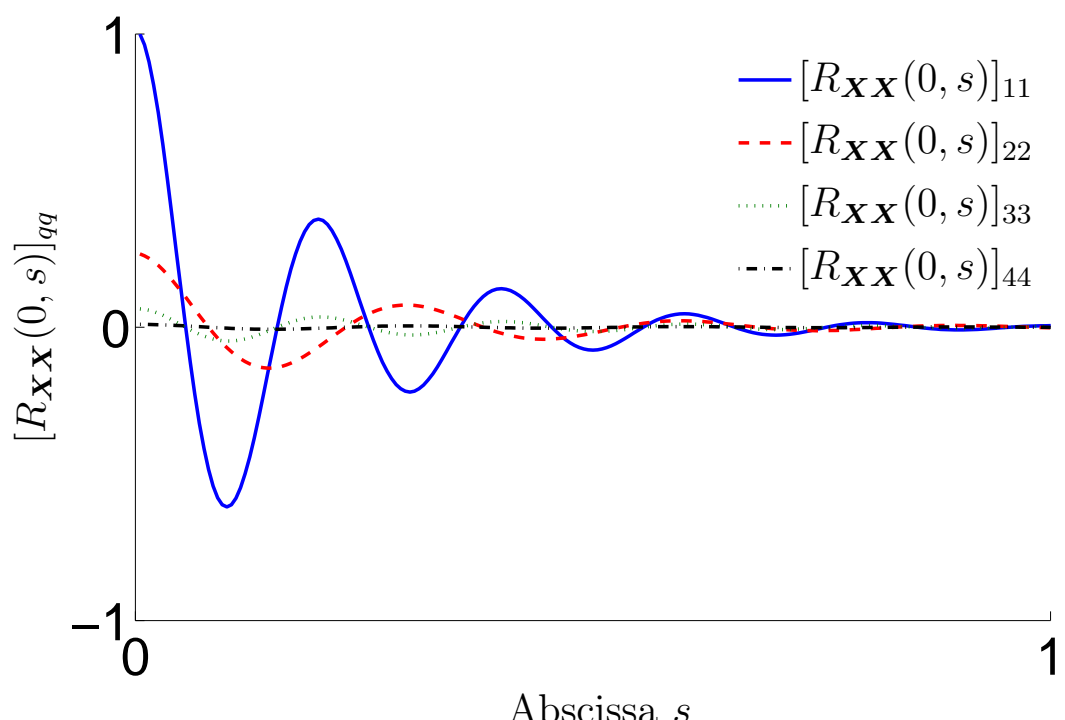

Figure 2: Representations of $s \mapsto\left[R_{\boldsymbol{X} \boldsymbol{X}}(0, s)\right]_{q q}$, for $1 \leq q \leq 4$.

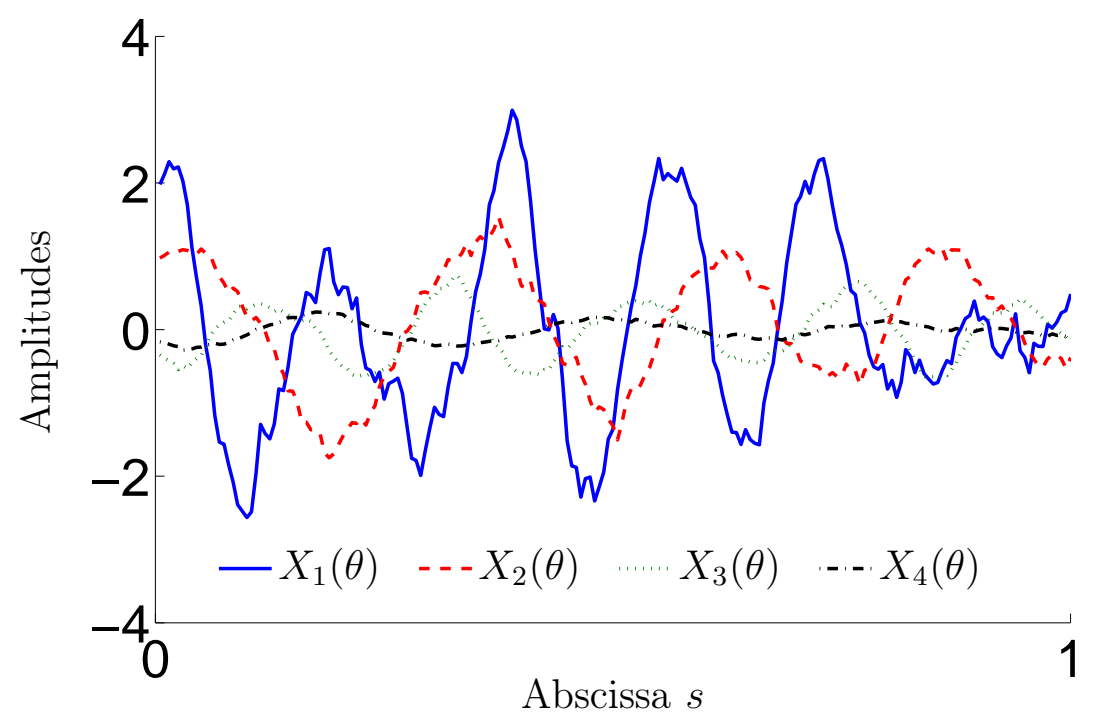

Figure 3: Representation of $\boldsymbol{X}(\theta)$. 


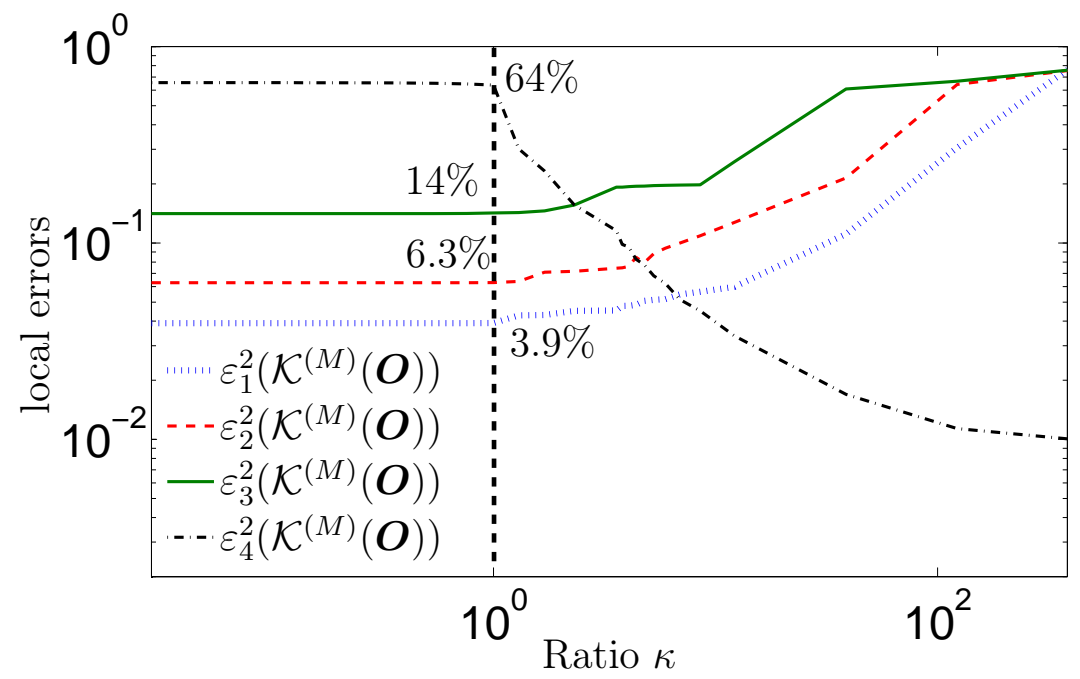

Figure 4: Comparison of local errors $\varepsilon_{q}^{2}\left(\mathcal{K}^{(M)}(\boldsymbol{O})\right)$, when $\boldsymbol{O}=$ $\frac{1}{\sqrt{3+\kappa^{2}}}(1,1,1, \kappa)$, with respect to $\kappa$, for $M=50$.

In the same manner, the results concerning the two dimensions case can be illustrated from this four dimensions case, by imposing:

$$
\boldsymbol{O}=\frac{1}{\sqrt{O_{1}^{2}+2.10^{-10}+O_{4}^{2}}}\left(O_{1}, 10^{-5}, 10^{-5}, O_{4}\right) .
$$

Indeed, in such a case, the weights of $X_{2}$ and $X_{3}$ will always be negligible. In Figure 5, it can therefore be seen that when ratio $O_{4} / O_{1}$ increases, $\varepsilon_{4}^{2}\left(\mathcal{K}^{(M)}(\boldsymbol{O})\right)$ decreases from its maximal value to its minimal value, whereas $\varepsilon_{1}^{2}\left(\mathcal{K}^{(M)}(\boldsymbol{O})\right)$ increases from its minimal value to its maximal value. As

$$
\left\{\begin{array}{l}
\lim _{O_{4}^{2} / O_{1}^{2} \rightarrow 0} \varepsilon_{1}^{2}\left(\mathcal{K}^{(M)}(\boldsymbol{O})\right)<\min _{2 \leq q \leq 4}\left\{\lim _{O_{4}^{2} / O_{1}^{2} \rightarrow 0} \varepsilon_{q}^{2}\left(\mathcal{K}^{(M)}(\boldsymbol{O})\right)\right\} \\
\lim _{O_{4}^{2} / O_{1}^{2} \rightarrow+\infty} \varepsilon_{4}^{2}\left(\mathcal{K}^{(M)}(\boldsymbol{O})\right)<\min _{1 \leq q \leq 3}\left\{\lim _{O_{4}^{2} / O_{1}^{2} \rightarrow 0} \varepsilon_{q}^{2}\left(\mathcal{K}^{(M)}(\boldsymbol{O})\right)\right\}
\end{array}\right.
$$

it exists a value for $O_{4} / O_{1}$ in $] 0,+\infty\left[\right.$ such that $\varepsilon_{1}^{2}$ and $\varepsilon_{4}^{2}$ are equal. This value allows us therefore to identify a projection family which is $M$-optimal for $\boldsymbol{X}$ with respect to the error $\max _{p \in\{1,4\}}\left\{\varepsilon_{q}^{2}\right\}$. 


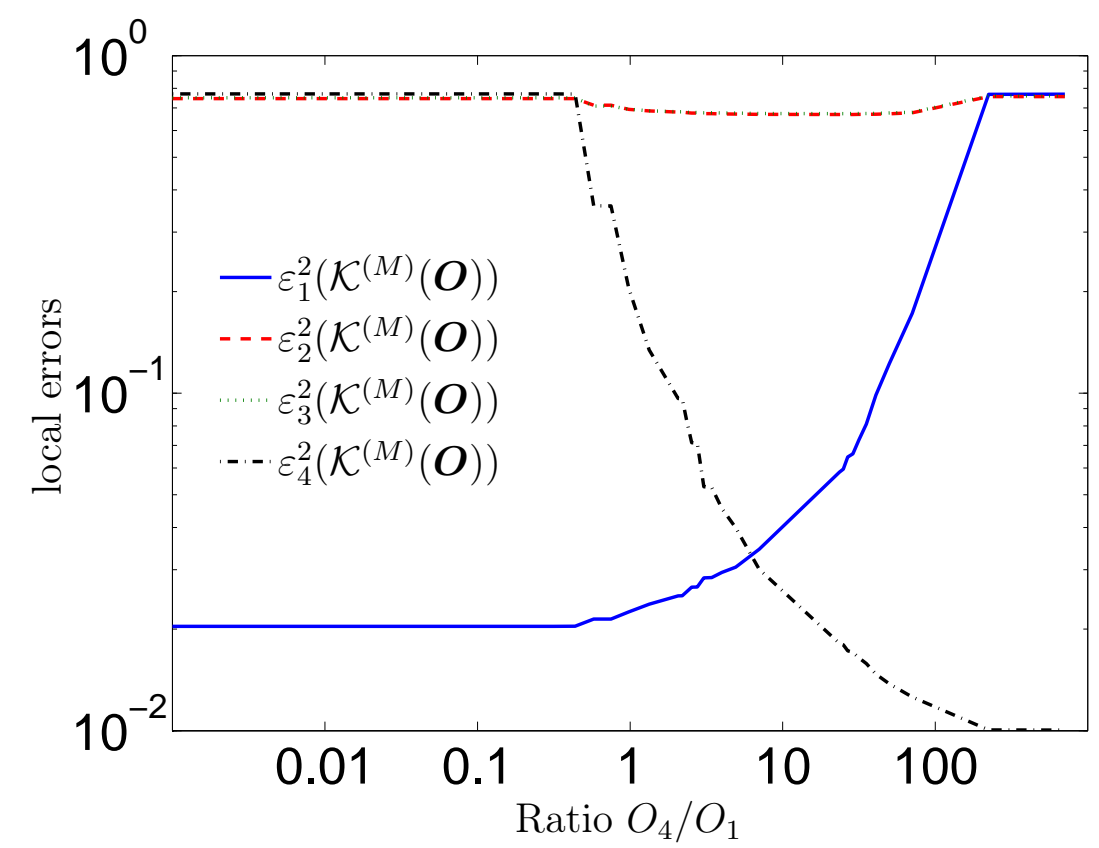

Figure 5: Comparison of local errors $\varepsilon_{q}^{2}\left(\mathcal{K}^{(M)}(\boldsymbol{O})\right)$, when $\boldsymbol{O}=$ $\frac{1}{\sqrt{O_{1}^{2}+2.10^{-10}+O_{4}^{2}}}\left(O_{1}, 10^{-5}, 10^{-5}, O_{4}\right)$ with respect to ratio $O_{4} / O_{1}$, for
$M=50$. 


\subsection{Identification of the optimal basis}

In Section 2, for all $\boldsymbol{\beta}$ in $\mathcal{S}^{(Q)}(1)$, optimal projection families $\mathcal{F}_{\boldsymbol{\beta}}^{(M)}$ and $\mathcal{F}_{\infty}^{(M)}$ have been introduced as the solutions of the two following optimization problems:

$$
\begin{aligned}
& \mathcal{F}_{\boldsymbol{\beta}}^{(M)}=\arg \min _{\mathcal{F}^{(M)} \in \mathbb{H}^{M}}\left\{\varepsilon_{\boldsymbol{\beta}}^{2}\left(\mathcal{F}^{(M)}\right)\right\}, \\
& \mathcal{F}_{\infty}^{(M)}=\arg \min _{\mathcal{F}^{(M)} \in \mathbb{H}^{M}}\left\{\varepsilon_{\infty}^{2}\left(\mathcal{F}^{(M)}\right)\right\} .
\end{aligned}
$$

In particular, for all $M \geq 1$, the choice

$$
\boldsymbol{\beta}=\left(\left\|X_{1}\right\|_{2},\left\|X_{2}\right\|_{2},\left\|X_{3}\right\|_{2},\left\|X_{4}\right\|_{2}\right)
$$

leads to the identification of the classical Karhunen-Loève family, which is called $\mathcal{F}_{L_{2}}^{(M)}$, for $\boldsymbol{X}$. The corresponding local errors, $\varepsilon_{q}^{2}\left(\mathcal{F}_{L_{2}}^{(M)}\right)$ can then be compared. For $M=50$, it can be seen in Figure 4 that $\varepsilon_{1}^{2}\left(\mathcal{F}_{L_{2}}^{(50)}\right)=3.9 \%$, $\varepsilon_{2}^{2}\left(\mathcal{F}_{L_{2}}^{(50)}\right)=6.3 \%, \varepsilon_{3}^{2}\left(\mathcal{F}_{L_{2}}^{(50)}\right)=14 \%$ and $\varepsilon_{4}^{2}\left(\mathcal{F}_{L_{2}}^{(50)}\right)=64 \%$. Due to the fact that $\left\|X_{1}\right\|_{2}>\left\|X_{2}\right\|_{2}>\left\|X_{3}\right\|_{2}>\left\|X_{4}\right\|_{2}$, it can thus be verified that the direct Karhunen-Loève expansion favorizes the description of component $X_{1}$, whereas component $X_{4}$ is not precisely characterized.

As explained in Section 2, other values for $\boldsymbol{\beta}$ have to be considered in order to improve the characterization of $X_{4}$. For instance, the choice $\boldsymbol{\beta}=$ $(0.5,0.5,0.5,0.5)$ corresponds to the minimization of the mean value of the local errors, $\varepsilon_{\mu}^{2}=\frac{1}{4} \sum_{q=1}^{4} \varepsilon_{q}^{2}$. Let $\mathcal{F}_{\mu}^{(M)}$ be the corresponding optimal family. Any other value for $\boldsymbol{\beta}$ can nevertheless be chosen. For instance, let $\mathcal{F}_{\boldsymbol{\beta}}^{(M)}$ be the $M$-optimal family corresponding to the case $\boldsymbol{\beta}=(0.1,2,1,0.5) / 2.2935$. At last, family $\mathcal{K}^{(M)}\left(\boldsymbol{O}_{\infty}^{(M)}\right)$ is introduced as the numerical solution of the algorithm defined by Eq. (46), with $\tau=10^{-3}$ and $\gamma=1 / 2$.

In this prospect, Figures 6 and 7 allow us to numerically illustrate that projection families $\mathcal{F}_{\boldsymbol{\beta}}^{(M)}, \mathcal{F}_{\mu}^{(M)}, \mathcal{F}_{L_{2}}^{(M)}$ and $\mathcal{K}^{(M)}\left(\boldsymbol{O}_{\infty}^{(M)}\right)$ can be identified from the scaled KL expansion, such that for any $M \geq 1$ :

- $\varepsilon_{\boldsymbol{\beta}}^{2}\left(\mathcal{F}_{\boldsymbol{\beta}}^{(M)}\right) \leq \min \left\{\varepsilon_{\boldsymbol{\beta}}^{2}\left(\mathcal{F}_{L_{2}}^{(M)}\right), \varepsilon_{\boldsymbol{\beta}}^{2}\left(\mathcal{K}^{(M)}\left(\boldsymbol{O}_{\infty}^{(M)}\right)\right), \varepsilon_{\boldsymbol{\beta}}^{2}\left(\mathcal{F}_{\mu}^{(M)}\right)\right\}$

- $\varepsilon_{\mu}^{2}\left(\mathcal{F}_{\mu}^{(M)}\right) \leq \min \left\{\varepsilon_{\mu}^{2}\left(\mathcal{F}_{L_{2}}^{(M)}\right), \varepsilon_{\mu}^{2}\left(\mathcal{K}^{(M)}\left(\boldsymbol{O}_{\infty}^{(M)}\right)\right), \varepsilon_{\mu}^{2}\left(\mathcal{F}_{\boldsymbol{\beta}}^{(M)}\right)\right\}$,

- $\varepsilon_{\infty}^{2}\left(\mathcal{K}^{(M)}\left(\boldsymbol{O}_{\infty}^{(M)}\right)\right) \leq \min \left\{\varepsilon_{\infty}^{2}\left(\mathcal{F}_{L_{2}}^{(M)}\right), \varepsilon_{\infty}^{2}\left(\mathcal{F}_{\boldsymbol{\beta}}^{(M)}\right), \varepsilon_{\infty}^{2}\left(\mathcal{F}_{\mu}^{(M)}\right)\right\}$, 
- $\varepsilon^{2}\left(\mathcal{F}_{L_{2}}^{(M)}\right) \leq \min \left\{\varepsilon^{2}\left(\mathcal{F}_{\boldsymbol{\beta}}^{(M)}\right), \varepsilon^{2}\left(\mathcal{K}^{(M)}\left(\boldsymbol{O}_{\infty}^{(M)}\right)\right), \varepsilon^{2}\left(\mathcal{F}_{\mu}^{(M)}\right)\right\}$

In particular, for $M=100$ :

$$
\begin{aligned}
& \left\{\begin{array}{l}
\varepsilon_{1}^{2}\left(\mathcal{F}_{L_{2}}^{(100)}\right)=1.7 \% \\
\varepsilon_{2}^{2}\left(\mathcal{F}_{L_{2}}^{(100)}\right)=3.0 \% \\
\varepsilon_{3}^{2}\left(\mathcal{F}_{L_{2}}^{(100)}\right)=5.8 \% \\
\varepsilon_{4}^{2}\left(\mathcal{F}_{L_{2}}^{(100)}\right)=17 \%
\end{array}, \quad\left\{\begin{array}{l}
\varepsilon_{1}^{2}\left(\mathcal{K}^{(100)}\left(\boldsymbol{O}_{\infty}^{(M)}\right)\right)=3.0 \% \\
\varepsilon_{2}^{2}\left(\mathcal{K}^{(100)}\left(\boldsymbol{O}_{\infty}^{(M)}\right)\right)=3.0 \% \\
\varepsilon_{3}^{2}\left(\mathcal{K}^{(100)}\left(\boldsymbol{O}_{\infty}^{(M)}\right)\right)=3.0 \% \\
\varepsilon_{4}^{2}\left(\mathcal{K}^{(100)}\left(\boldsymbol{O}_{\infty}^{(M)}\right)\right)=3.0 \%
\end{array},\right.\right. \\
& \left\{\begin{array}{l}
\varepsilon^{2}\left(\mathcal{F}_{L_{2}}^{(100)}\right)=2.3 \% \\
\varepsilon^{2}\left(\mathcal{K}^{(100)}\left(\boldsymbol{O}_{\infty}^{(M)}\right)\right)=3.0 \% \\
\varepsilon_{\infty}^{2}\left(\mathcal{F}_{L_{2}}^{(100)}\right)=17 \% \\
\varepsilon_{\infty}^{2}\left(\mathcal{K}^{(100)}\left(\boldsymbol{O}_{\infty}^{(M)}\right)\right)=3.0 \%
\end{array} .\right.
\end{aligned}
$$

Whereas family $\mathcal{F}_{L_{2}}^{(100)}$ can defavorize the description of a particular component of $\boldsymbol{X}$ to minimize $\varepsilon^{2}$, family $\mathcal{K}^{(100)}\left(\boldsymbol{O}_{\infty}^{(M)}\right)$ tries to equilibrate the precision of the description of each component. To do so, the local error of some components can increase to make the other decrease. Indeed, in this example, $\varepsilon_{1}^{2}\left(\mathcal{F}_{L_{2}}^{(100)}\right)<\varepsilon_{1}^{2}\left(\mathcal{K}^{(100)}\left(\boldsymbol{O}_{\infty}^{(M)}\right)\right)$ whereas $\varepsilon_{4}^{2}\left(\mathcal{F}_{L_{2}}^{(100)}\right)>\varepsilon_{4}^{2}\left(\mathcal{K}^{(100)}\left(\boldsymbol{O}_{\infty}^{(M)}\right)\right)$. From Eq. (38), it can moreover be seen that in this case:

$$
\left|\varepsilon_{\infty}^{2}\left(\mathcal{F}_{\infty}^{(100)}\right)-\varepsilon_{\infty}^{2}\left(\mathcal{K}^{(100)}\left(\boldsymbol{O}_{\infty}^{(M)}\right)\right)\right| \leq \tau=0.1 \% .
$$

\section{Conclusions}

In spite of the increasing computational power that has encouraged the development of computational models with always more degrees of freedom, reduction methods, such as the Karhunen-Loève expansion, still have a big role to play to make the solving of these problems faster and robuster. When dealing with $\mathbb{R}^{Q}$-valued random fields $\boldsymbol{X}=\left(X_{1}, \cdots, X_{Q}\right)$, it has however been shown in this work that the direct truncated KL expansion, which minimizes the total mean-squared error, tends to better characterize the components of $\boldsymbol{X}$ that have the highest signal energy. In this context, a particular adaptation of the KL expansion has been introduced in this paper. Based on a scaling transformation of $\boldsymbol{X}$, this original decomposition allows defining projection basis that can favorize or defavorize on purpose the characterization of a particular component of $\boldsymbol{X}$. This expansion appears to be also 

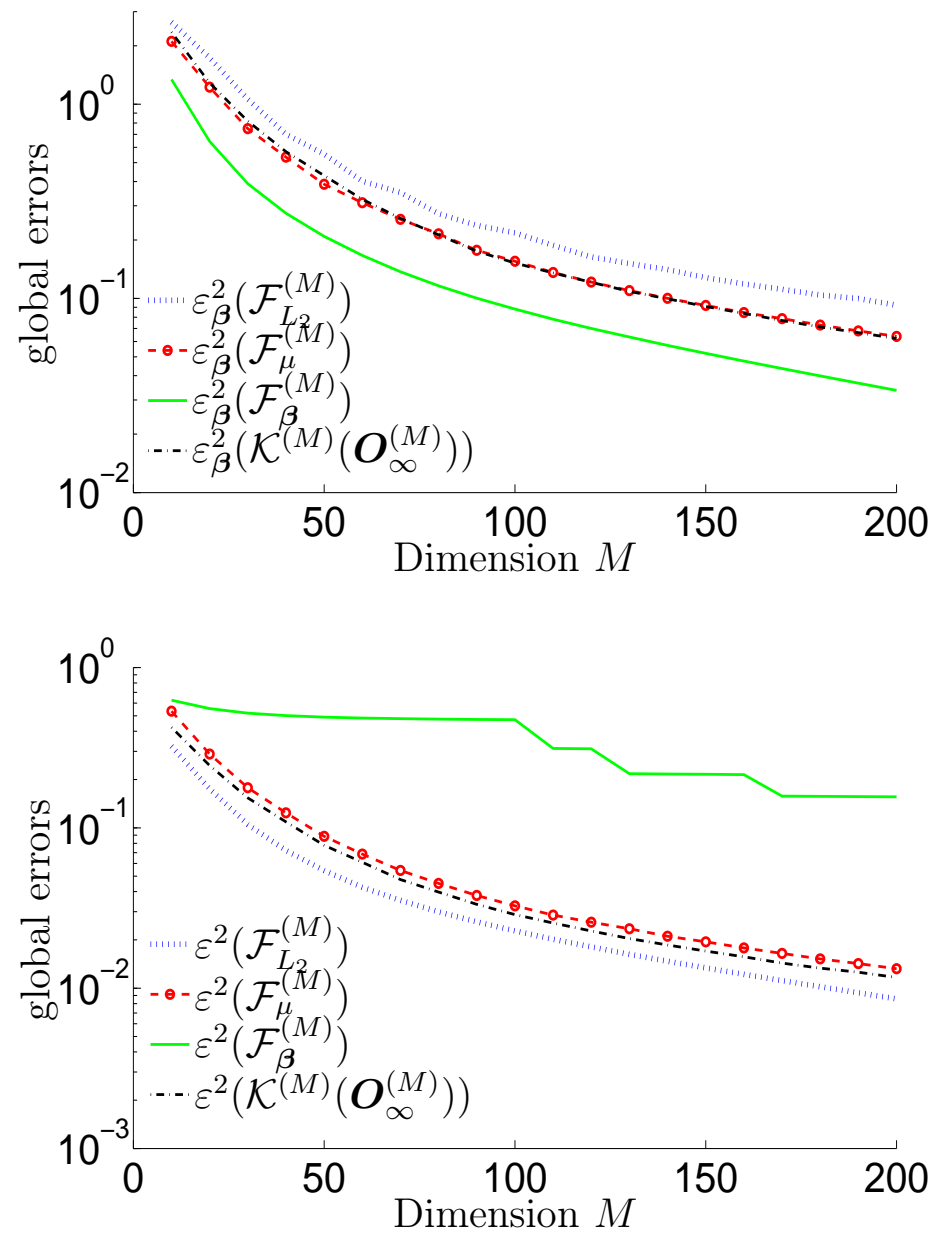

Figure 6: Evolution of errors $\varepsilon_{\boldsymbol{\beta}}^{2}$ and $\varepsilon^{2}$ with respect to the dimension of the projection family, $M$. 

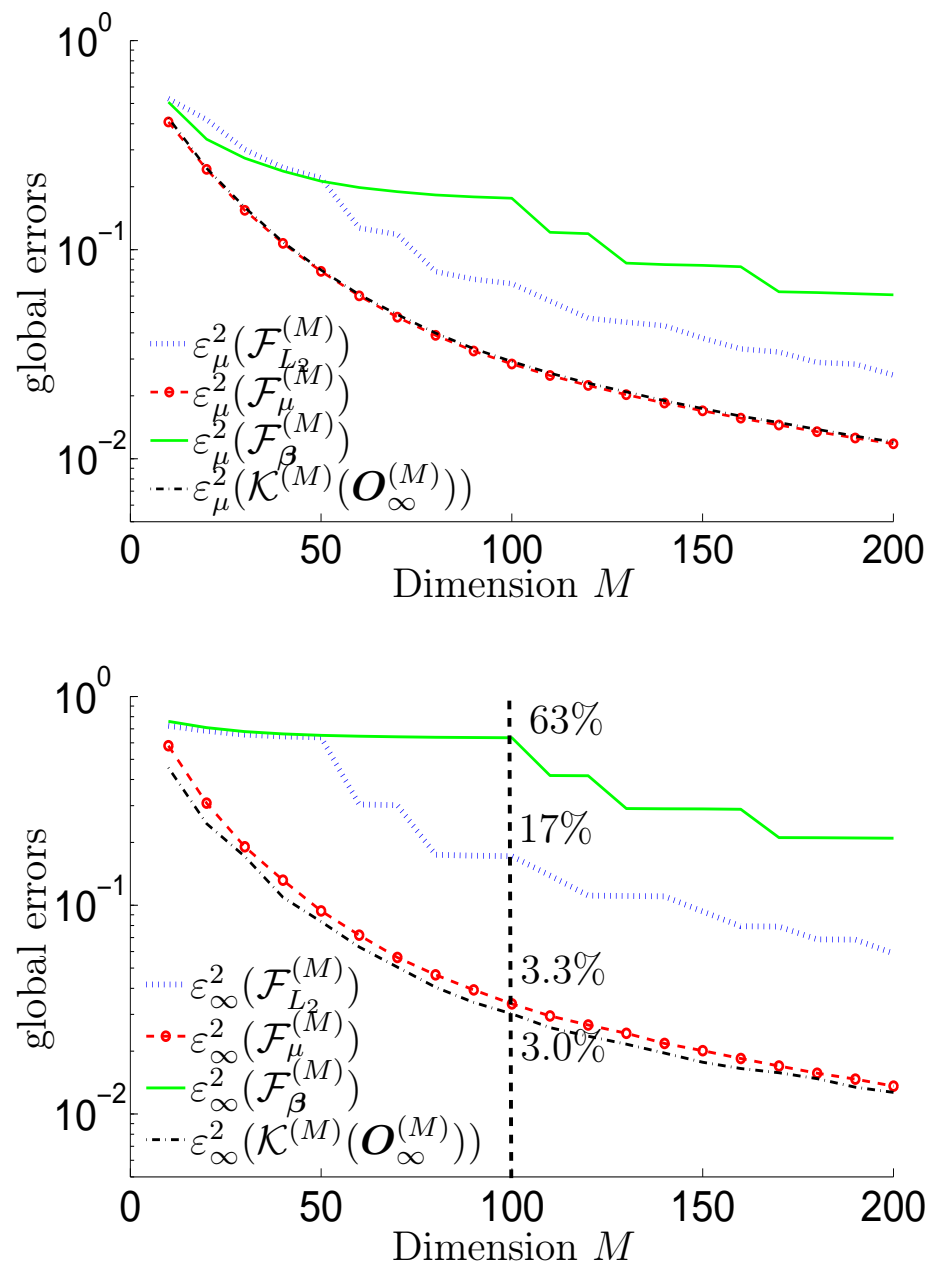

Figure 7: Evolution of errors $\varepsilon_{\mu}^{2}$ and $\varepsilon_{\infty}^{2}$ with respect to the dimension of the projection family, $M$. 
very relevant to identify projection basis that minimize the maximal value of the local errors of $\boldsymbol{X}$. Finally, when interested in studying complex systems that are excited by vector-valued random fields (one can think about the interactions between trains and track irregularities, buildings and earthquakes, harbors and swell, etc.), the method proposed opens new opportunities to adapt the projection basis with respect to the quantities of interest of the systems.

\section{Acknowledgments}

This work was supported by SNCF (Innovation and Research Department).

\section{AppendixA. Proof of Lemma 2}

Using the notations of Section $2,\left\{\boldsymbol{k}^{i}(\boldsymbol{O}), i \geq 1\right\}$ defines a spatially orthonormal basis of $\mathcal{P}(\Omega)$. Autocorrelation function $\left[R_{\boldsymbol{Y} \boldsymbol{Y}}(\boldsymbol{O})\right]$ can therefore be projected on this basis, such that, by construction of the Karhunen-Loève basis:

$$
\left[R_{\boldsymbol{Y} \boldsymbol{Y}}(\boldsymbol{O})\right]=\sum_{i \geq 1} \lambda_{i}(\boldsymbol{O}) \boldsymbol{k}^{i}(\boldsymbol{O}) \otimes \boldsymbol{k}^{i}(\boldsymbol{O})
$$

Let $\mathcal{B}=\left\{\boldsymbol{b}^{i}, 1 \leq i\right\}$ be another countable basis of Hilbertian space $\mathcal{P}(\Omega)$, and $\mathcal{F}^{(M)}=\left\{\boldsymbol{b}^{i}, 1 \leq i \leq M\right\}$ be a $M$-dimension subset of $\mathcal{B}$. For all $i \geq 1$, $\boldsymbol{f}^{i}$ can then be projected on $\left\{\boldsymbol{k}^{i}(\boldsymbol{O}), i \geq 1\right\}$ :

$$
\boldsymbol{f}^{i}=\sum_{j \geq 1} P_{i j} \boldsymbol{k}^{j}(\boldsymbol{O}), \quad P_{i j}=\left(\boldsymbol{f}^{i}, \boldsymbol{k}^{j}(\boldsymbol{O})\right) .
$$

Without loss of generality, familly $\mathcal{F}$ can be supposed to be spatially orthonormal, as it can be orthonormalized a posteriori without modifying the corresponding projection error. From Eqs. (201), this yields:

$$
1=\left(\boldsymbol{f}^{i}, \boldsymbol{f}^{i}\right)=\sum_{j \geq 1} \sum_{\ell \geq 1} P_{i j} P_{i \ell}\left(\boldsymbol{k}^{j}(\boldsymbol{O}), \boldsymbol{k}^{\ell}(\boldsymbol{O})\right)=\sum_{j \geq 1} P_{i j}^{2}
$$

Let $\tilde{\boldsymbol{Y}}^{(M)}$ be the projection of random field $\boldsymbol{Y}=[\operatorname{Diag}(\boldsymbol{O})] \boldsymbol{X}$ on $\mathcal{F}^{(M)}$ : 


$$
\tilde{\boldsymbol{Y}}^{(M)}=\sum_{i=1}^{M} \boldsymbol{f}^{i} C_{i}, \quad C_{i}=\left(\boldsymbol{Y}, \boldsymbol{f}^{i}\right) .
$$

Random field $\widetilde{\boldsymbol{X}}^{(M)}$ is thus introduced as:

$$
\widetilde{\boldsymbol{X}}^{(M)}=[\operatorname{Diag}(\boldsymbol{O})]^{-1} \widetilde{\boldsymbol{Y}}^{(M)} .
$$

From Eqs. (A.1) and (A.4), we get, for all $M \geq i \geq 1$ :

$$
E\left[C_{i}^{2}\right]=\int_{\Omega^{2}}\left(\boldsymbol{f}^{i}(s)\right)^{T}\left[R_{\boldsymbol{Y} \boldsymbol{Y}}\left(\boldsymbol{O}, s, s^{\prime}\right)\right] \boldsymbol{f}^{i}\left(s^{\prime}\right) d s d s^{\prime}=\sum_{j \geq 1} \lambda_{j}(\boldsymbol{O}) P_{i j}^{2}
$$

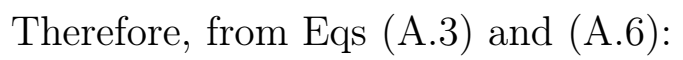

$$
\begin{aligned}
\sum_{i=1}^{M}\left(\lambda_{i}(\boldsymbol{O})-E\left[C_{i}^{2}\right]\right) & =\sum_{i=1}^{M} \lambda_{i}(\boldsymbol{O}) \sum_{j \geq 1} P_{i j}^{2}-\sum_{i=1}^{M} \sum_{j \geq 1} \lambda_{j}(\boldsymbol{O}) P_{i j}^{2} \\
& =\sum_{i=1}^{M} \sum_{j \geq 1} P_{i j}^{2}\left(\lambda_{i}(\boldsymbol{O})-\lambda_{j}(\boldsymbol{O})\right) \\
& =\sum_{i=1}^{M} \sum_{j \geq M+1} P_{i j}^{2}\left(\lambda_{i}(\boldsymbol{O})-\lambda_{j}(\boldsymbol{O})\right) \\
& \geq\left(\lambda_{M}(\boldsymbol{O})-\lambda_{M+1}(\boldsymbol{O})\right) \sum_{i=1}^{M} \sum_{j \geq M+1} P_{i j}^{2} \geq 0
\end{aligned}
$$

as by construction, for all $j \geq i, \lambda_{j}(\boldsymbol{O}) \leq \lambda_{i}(\boldsymbol{O})$. Moreover, it can be noticed that, by definition of matrix $[\operatorname{Diag}(\boldsymbol{O})]$, for $1 \leq q \leq Q$ :

$$
\begin{aligned}
& E\left[\left(X_{q}-\widehat{X}_{q}^{(M)}, X_{q}-\widehat{X}_{q}^{(M)}\right)\right]=E\left[\left(O_{q}^{-1}\left(Y_{q}-\widehat{Y}_{q}^{(M)}\right), O_{q}^{-1}\left(Y_{q}-\widehat{Y}_{q}^{(M)}\right)\right)\right] \\
& =O_{q}^{-2} \sum_{M+1 \leq i} \lambda_{i}(\boldsymbol{O})\left(k_{q}^{i}(\boldsymbol{O}), k_{q}^{i}(\boldsymbol{O})\right)
\end{aligned}
$$


where it is reminded that $\widehat{\boldsymbol{Y}}^{(M)}$ is the projection of $\boldsymbol{Y}=[\operatorname{Diag}(\boldsymbol{O})] \boldsymbol{X}$ on $\mathcal{K}^{(M)}(\boldsymbol{O})$ and $\widehat{\boldsymbol{X}}^{(M)}=[\operatorname{Diag}(\boldsymbol{O})]^{-1} \widehat{\boldsymbol{Y}}^{(M)}$. In the same manner:

$$
\begin{aligned}
& E\left[\left(X_{q}-\tilde{X}_{q}^{(M)}, X_{q}-\tilde{X}_{q}^{(M)}\right)\right]=E\left[\left(O_{q}^{-1}\left(Y_{q}-\tilde{Y}_{q}^{(M)}\right), O_{q}^{-1}\left(Y_{q}-\tilde{Y}_{q}^{(M)}\right)\right)\right] \\
& =O_{q}^{-2} \sum_{M+1 \leq i} E\left[C_{i}^{2}\right]\left(f_{q}^{i}, f_{q}^{i}\right)
\end{aligned}
$$

It can finally be deduced from Eqs. (A.7), (A.8) and (A.9) that:

$$
\begin{aligned}
& \sum_{q=1}^{Q} O_{q}^{2}\left\|X_{q}\right\|_{2}^{2} \varepsilon_{q}^{2}\left(\mathcal{K}^{(M)}(\boldsymbol{O})\right)-\sum_{q=1}^{Q} O_{q}^{2}\left\|X_{q}\right\|_{2}^{2} \varepsilon_{q}^{2}\left(\mathcal{F}^{(M)}\right) \\
& =\sum_{q=1}^{Q} O_{q}^{2}\left[E\left[\left(X_{q}-\widehat{X}_{q}^{(M)}, X_{q}-\widehat{X}_{q}^{(M)}\right)\right]-E\left[\left(X_{q}-\widetilde{X}_{q}^{(M)}, X_{q}-\widetilde{X}_{q}^{(M)}\right)\right]\right] \\
& =\sum_{M+1 \leq i}\left[\lambda_{i}(\boldsymbol{O}) \sum_{q=1}^{Q}\left(k_{q}^{i}(\boldsymbol{O}), k_{q}^{i}(\boldsymbol{O})\right)-E\left[C_{i}^{2}\right] \sum_{q=1}^{Q}\left(f_{q}^{i}, f_{q}^{i}\right)\right] \\
& =\sum_{M+1 \leq i}\left[\lambda_{i}(\boldsymbol{O})-E\left[C_{i}^{2}\right]\right] \\
& =\sum_{i=1}^{M}\left[E\left[C_{i}^{2}\right]-\lambda_{i}(\boldsymbol{O})\right] \\
& \leq 0 .
\end{aligned}
$$

This result being true for all family $\mathcal{F}^{(M)}$ in $\mathbb{H}^{M}$, family $\mathcal{K}^{(M)}(\boldsymbol{O})$ is thus $M$-optimal for $\boldsymbol{X}$ regarding error $\sum_{q=1}^{Q} O_{q}^{2}\left\|X_{q}\right\|_{2}^{2} \varepsilon_{q}^{2}$.

\section{AppendixB. Generation of the matrix-valued autocorrelation ma- trix}

For $1 \leq p, q \leq 4$, matrix-valued autocorrelation function $\left[R_{\boldsymbol{X} \boldsymbol{X}}\right]$ is chosen such that: 


$$
\left[R_{\boldsymbol{X} \boldsymbol{X}}\left(s, s^{\prime}\right)\right]_{p q}=\frac{c_{p} c_{q}\left(1+\delta_{p q}\right)}{2} \sum_{k=1}^{200} \sqrt{\lambda_{k}^{(p)} \lambda_{k}^{(q)}} d_{k}^{(p)}(s) d_{k}^{(q)}\left(s^{\prime}\right), \forall\left(s, s^{\prime}\right) \in[0,1]^{2},
$$

where for all $1 \leq k \leq 200$ :

$$
\begin{gathered}
\int_{0}^{1} h_{p}\left(s, s^{\prime}\right) d_{k}^{(p)}(s) d s=\lambda_{k}^{(p)} d_{k}^{(p)}\left(s^{\prime}\right), \\
h_{p}\left(s, s^{\prime}\right)=\exp \left(-\left|s-s^{\prime}\right| / \ell_{p}\right) \cos \left(\omega_{p}\left|s-s^{\prime}\right|\right) \cos \left(T_{p} s\right), \\
\lambda_{k}^{(p)} \geq \lambda_{k+1}^{(p)}>0, \\
\left(d_{k}^{(p)}, d_{k}^{(q)}\right)=\delta_{p q} .
\end{gathered}
$$

The numerical values of vectors $\boldsymbol{c}=\left(c_{1}, \cdots, c_{4}\right), \boldsymbol{\omega}=\left(\omega_{1}, \cdots, \omega_{4}\right), \boldsymbol{\ell}=$ $\left(\ell_{1}, \cdots, \ell_{4}\right), \boldsymbol{T}=\left(T_{1}, \cdots, T_{4}\right)$ are gathered in Figure B.1, Several comments can be made about this formalism.

- Application $\left(s, s^{\prime}\right) \mapsto h_{q}\left(s, s^{\prime}\right)$ is not necessary positive-definite regarding the chosen numerical parameters, but only its 200 highest strictly positive eigenvalues, $\left\{\lambda_{k}^{(q)}, 1 \leq k \leq 200\right\}$, are considered.

- Couples $\left\{\lambda_{k}^{(q)}, d_{k}^{(q)}\right\}$ are solutions of the Fredholm problem associated with $h_{q}$, but are not solutions of the Fredholm problem associated with $\left[R_{\boldsymbol{X} \boldsymbol{X}}\right]$.

- Coefficient $c_{q}^{2}$ can be related to the signal energy of $X_{q}$, such that if $c_{p}>c_{q},\left\|X_{p}\right\|_{2}^{2}>\left\|X_{q}\right\|_{2}^{2}$.

- Coefficient $2 \pi / \omega_{q}$ can be considered as a pseudo-wavelength for the mean-squared stationnary part of $\left[R_{\boldsymbol{X} \boldsymbol{X}}\right]_{p q}$.

- Coefficient $\ell_{q}$ can be seen as the auto-correlation length of $X_{q}$.

- Coefficient $T_{q}$ is introduced as a perturbation for $\left[R_{\boldsymbol{X} \boldsymbol{X}}\right]_{p q}$, such that the smaller $T_{q}$ is, the less mean-squared stationnary $\left[R_{\boldsymbol{X} \boldsymbol{X}}\right]_{p q}$ is. 


\begin{tabular}{|c|c|c|c|c|}
\hline $\mathrm{q}$ & $c_{q}$ & $\omega_{q} S /(2 \pi)$ & $\ell_{q} / S$ & $T_{q} S /(2 \pi)$ \\
\hline 1 & 1 & $20 \%$ & $20 \%$ & 5 \\
2 & 0.5 & $30 \%$ & $25 \%$ & 7 \\
3 & 0.25 & $20 \%$ & $35 \%$ & 8 \\
4 & 0.1 & $30 \%$ & $40 \%$ & 10 \\
\hline
\end{tabular}

Table B.1: Numerical values used in the definition of autocorrelation matrix $\left[R_{\boldsymbol{X} \boldsymbol{X}}\right]$

[1] C. Allery, A. Hambouni, D. Ryckelynck, N. Verdon, A priori reduction method for solving the two-dimensional burgers' equations, Applied Mathematics and Computation 217 (2011) 6671-6679.

[2] J. Atwell, B. King, Proper orthogonal decomposition for reduced basis feedback controllers for parabolic equations, Math. Comput. Modell. 33 (1-3) (2001) 1-19.

[3] G. Berkooz, P. Holmes, J. Lumley, The proper orthogonal decomposition in the analysis of turbulent flows, Annu. Rev. Fluid Mech. 25 (1993) $539-575$.

[4] G. P. Brooks, J. M. Powers, A karhunen-loève least-squares technique for optimization of geometry of a blunt body in supersonic flow, Journal of Computational Physics 195 (2004) 387-412.

[5] E. Christensen, M. Brons, J. Sorensen, Evaluation of proper orthogonal decomposition-based decomposition techniques applied to parameter dependent nonturbulent flows, SIAM J. Sci. Comput 21 (4) (2000) $1419-1434$.

[6] S. Huang, S. Quek, K. Phoon, Convergence study of the truncated karhunen-loève expansion for simulation of stochastic processes, Int J Num Meth Engng 52 (9) (2001) 1029-43.

[7] K. Kunisch, S. Volkwein, Galerkin proper orthogonal decomposition methods for parabolic problems, Numer. Math. 90 (1) (2001) 117-148.

[8] L. Li, K. Phoon, S. Quek, Comparison between karhunen-loève expansion and translation-based simulation of non-gaussian processes, Computers and Structures 85 (2007) 264-76. 
[9] X. Ma, N. Zabaras, Kernel principal component analysis for stochastic input model generation, Comptes rendus de l'Académie des sciences de Paris 220 (1945).

[10] A. Nouy, O. L. Maître, Generalized spectral decomposition method for stochastic non-linear problems, J. Comput. Phys. 228 (1) (2009) 202235 .

[11] K. Phoon, S. Huang, S. Quek, Implementation of karhunen-loeve expansion for simulation using a wavelet-galerkin scheme, Probabilistic Engineering Mechanics 17 (2002) 293-303.

[12] K. Phoon, S. Huang, S. Quek, Simulation of strongly non-gaussian processes using karhunen-loeve expansion, Probabilistic Engineering Mechanics 20 (2005) 188-198.

[13] C. Schwab, R. A. Todor, Karhunen-loeve approximation of random fields by generalized fast multipole methods, Journal of Computational Physics 217 (2006) 100-122.

[14] P. Spanos, B. Zeldin, Galerkin sampling method for stochastic mechanics problems, Journal of Engineering Mechanics 120 (5) (1994) 10911106.

[15] P. Spanos, M. Beer, J. Red-Horse, Karhunen -loève expansion of stochastic processes with a modified exponential covariance kernel, Journal of Engineering Mechanics 133 (7) (2007) 773-779.

[16] B. Wen, N. Zabaras, A multiscale approach for model reduction of random microstructures, Computational Materials Science 63 (2012) 269-285.

[17] M. Williams, The eigenfunctions of the karhunen-loeve integral equation for a spherical system, Propabilistic Engineering Mechanics 26 (2011) 202-207.

[18] S. Q. Wu, S. S. Law, Statistical moving load identification including uncertainty, Probabilistic Engineering Mechanics 29 (2012) 70-78.

[19] M. Arnst, R. Ghanem, C. Soize, Identification of bayesian posteriors for coefficients of chaos expansions, Journal of Computational Physics 229 (9) (2010) 3134-3154. 
[20] S. Das, R. Ghanem, S. Finette, Polynomial chaos representation of spatio-temporal random field from experimental measurements, J. Comput. Phys. 228 (2009) 8726-8751.

[21] Y. M. Marzouk, H. N. Najm, Dimensionality reduction and polynomial chaos acceleration of bayesian inference in inverse problems, J. Comput. Phys. 228 (2009) 1862-1902.

[22] H. G. Matthies, C. Bucher, Finite elements for stochastic media problems, Comput. Methods Appli. Mech. Engrg. 168 (1999) 3-17.

[23] H. G. Matthies, A. Keese, Galerkin methods for linear and nonlinear elliptic stochastic partial differential equations, Comput. Methods Appli. Mech. Engrg. 194 (2005) 1295-1331.

[24] G. Perrin, C. Soize, D. Duhamel, C. Funfschilling, Identification of polynomial chaos representations in high dimension from a set of realizations, SIAM - Journal of Scientific Computing (accepted 2012).

[25] S. Sakamoto, R. Ghanem, Polynomial chaos decomposition for the simulation of non-gaussian non stationary stochastic processes, J. Engrg. Mechanics 128 (2002) 190-201.

[26] S. Sakamoto, R. Ghanem, Simulation of multi-dimensional non-gaussian non-stationary random fields, Probabilistic Engineering Mechanics 17 (2002) 167-176.

[27] C. Soize, R. Ghanem, Physical systems with random uncertainties: Chaos representations with arbitrary probability measure, SIAM J. Sci. Comput. 26 (2004).

[28] C. Soize, Generalized probabilistic approach of uncertainties in computational dynamics using random matrices and polynomial chaos decompositions., Internat. J. Numer. Methods Engrg. 81 (2010) 939-970.

[29] D. Zhang, Z. Lu, An efficient, high-order perturbation approach for flow in random porous media via karhunen-loeve and polynomial expansions, Journal of Computational Physics 194 (2) (2004) 773-794.

[30] O. Le Maître, O. Knio, Spectral Methods for Uncertainty Quantification, Springer, 2010. 
[31] P. Hansen, Numerical tools for analysis and solution of fredholm integral-equations of the 1st kind, Inverse problems 8 (6) (1992) 849872.

[32] J. Weese, A reliable and fast method for the solution of fredholm integral-equations of the 1st kind based on tikhonov regularization, Computer physics communications 69 (1992) 99-111. 